\title{
DISCOVERY AND CHARACTERIZATION OF WIDE BINARY SYSTEMS WITH A VERY LOW MASS COMPONENT
}

\author{
Frédérique Baron ${ }^{1}$, David Lafrenière ${ }^{1}$, Étienne Artigau ${ }^{1}$, René Doyon ${ }^{1}$, Jonathan Gagné ${ }^{1}$, \\ Cassy L. Davison ${ }^{2}$, Lison Malo ${ }^{3}$, Jasmin Robert ${ }^{1}$, Daniel Nadeau ${ }^{1}$, and Céline Reylé ${ }^{4}$ \\ ${ }^{1}$ Département de Physique, Université de Montréal, C.P. 6128 Succ. Centre-ville, Montréal, Qc H3C 3J7, \\ Canada \\ 2 Department of Physics and Astronomy, Georgia State University, Atlanta, GA 30303, USA \\ ${ }^{3}$ Canada-France-Hawaii Telescope, 65-1238 Mamalahoa Hwy, Kamuela, HI 96743, USA \\ ${ }^{4}$ Institut Utinam, CNRS UMR6213, Université de Franche-Comté, OSU THETA \\ Franche-Comté-Bourgogne, Observatoire de Besan[c]on, BP 1615, 25010 Besanon Cedex, France
}

\begin{abstract}
We report the discovery of 14 low-mass binary systems containing mid-M to mid-L dwarf companions with separations larger than $250 \mathrm{AU}$. We also report the independent discovery of 9 other systems with similar characteristics that were recently discovered in other studies. We have identified these systems by searching for common proper motion sources in the vicinity of known high proper motion stars, based on a cross-correlation of wide area near-infrared surveys (2MASS, SDSS, and SIMP). An astrometric follow-up, for common proper motion confirmation, was made with SIMON and/or CPAPIR at the OMM $1.6 \mathrm{~m}$ and CTIO $1.5 \mathrm{~m}$ telescopes for all the candidates identified. A spectroscopic follow-up was also made with GMOS or GNIRS at Gemini to determine the spectral types of 11 of our newly identified companions and 10 of our primaries. Statistical arguments are provided to show that all of the systems we report here are very likely to be physical binaries. One of the new systems reported features a brown dwarf companion: LSPM J1259+1001 (M5) has an L4.5 (2M1259+1001) companion at 340 AU. This brown dwarf was previously unknown. Seven other systems have a companion of spectral type L0-L1 at a separation in the 250-7500 AU range. Our sample includes 14 systems with a mass ratio below 0.3 .
\end{abstract}

Subject headings: binaries: general — stars: low-mass, brown dwarfs

\section{INTRODUCTION}

In the last two decades, the search for lowmass stars and brown dwarfs has intensified with the advent of wide area surveys such as 2MASS (Cutri et al. 2003), DENIS (Epchtein et al. 1999), SDSS (Ahn et al. 2012), UKIDSS (Lawrence et al. 2007), and more recently WISE (Cutri \& et al. 2012). In addition, a handful of surveys were aimed at finding nearby binary systems including low mass stars such as the surveys of Reid \& Gizis (1997) and Reid et al. (2001) for ex-

\footnotetext{
${ }^{1}$ baron@astro.umontreal.ca
}

ample. Dhital et al. (2010) assembled the SLoWPoKES catalog which contains 1342 binary systems with a projected separation of $\gtrsim 500 \mathrm{AU}$, in which one of the components is a mid-K to mid$\mathrm{M}$ dwarf. Numerous wide binary systems have also been discovered individually. Of all the systems known, very few have a separation larger than 7000 AU. The system composed of NLTT 20346 (an M5V and M6V binary) and 2MASS J0850359+105716 (an L6 dwarf) is one of these, with a separation of 7700 AU (Radigan et al. 2009), while Konigstuhl 3 A-BC (Faherty et al. 2011), a hierarchical system composed of an F8V orbited by an M8V and L3 binary, has a separa- 
tion of $12000 \mathrm{AU}$.

An interest of binary systems is that both components share the same age and metallicity. For systems comprising an $\mathrm{M}$ dwarf companion for example, this can help calibrate the metallicity scale for this type of star (Bonfils et al. 2005). The components of wide visual binaries can also be studied in detail individually using seeing-limited instruments, making their characterization much easier. Additionally, the evolution of each component of a wide binary system has not been influenced by the other component, and both can be viewed as an "isolated" star.

The discovery of numerous low-mass stars and brown dwarfs has led to a growing interest in understanding the formation mechanism of these objects whose masses are below the typical Jeans mass in a molecular cloud (Meyer et al. 2000), and numerous hypotheses about their origin have been formulated over the years. Binary systems containing a low mass component provide a good tool for attempting to discriminate between the various possibilities, as their properties (e.g., separations, mass ratios) may be affected differently by different processes (Duchêne \& Kraus 2013). Wide binary systems with separation larger than hundreds of AU are of particular interest for this purpose as, being more weakly gravitationally bound and spanning sizes larger than typical protostellar disks, they bring additional and perhaps more stringent constraints on the underlying formation processes.

In this article, we report the discovery of 14 new binary systems that all have a component with a spectral type later than M6 and a separation larger than $250 \mathrm{AU}$. We also recovered nine previously known binaries with similar characteristics. Section 2 details our archival search for wide binaries and the identification of our candidates, while Section 3 describes the follow-up photometry and the calculations of the proper motion. The spectroscopic follow-up as well as the results extracted from these data are explained in Section 4. The probability of random alignement has been calculated for each system and is presented in Section 5 A short discussion is presented in section 6. Our results are summarized in section 7

\section{SEARCH AND IDENTIFICATION OF THE CANDIDATE COMPAN- IONS}

\subsection{Sample}

We searched for new binary systems comprising a low-mass component through common proper motion based on multi-epoch wide area nearinfrared surveys. As a starting point for potential primary stars, we considered the NLTT (Salim \& Gould 2003) and LSPM (Lépine 2005) catalogs which include stars with proper motions larger than 0.18 mas $\mathrm{yr}^{-1}$ and 0.15 mas $\mathrm{yr}^{-1}$, respectively. We retained only the stars with a relative proper motion measurement error of less than $30 \%$. The large and precise proper motions of these stars are useful to limit the number of false positives, i.e., common proper motion between two sources resulting from chance or measurement errors. To calculate the proper motion of the sources in the vicinity of these target stars, we compared the 2MASS PSC data (Cutri et al. 2003) to data from the SDSS (Adelman-McCarthy et al. 2008) and/or the SIMP (Artigau et al. 2009) surveys. Of the roughly 60,000 stars included in the NLTT and LSPM catalogs, about 25,000 have accurate proper motion measurements and were observed by SIMP or SDSS; those constitute our initial sample. Target baseline's between the SDSS/SIMP and 2MASS observations span from 1 to 11 years, and the typical uncertainty of the calculated proper motions was 40 mas $\mathrm{yr}^{-1}$.

\subsection{Identifying Companions}

For each target star, we calculated the proper motion of all sources within a radius of $4^{\prime}$ and selected any source with a proper motion within

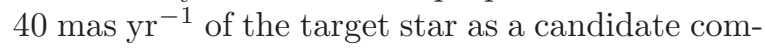
panion. Visual inspection was performed for all candidates to reject false detections of artifacts that are identified within catalogs as point sources. The available photometry from the 2MASS PSC catalog was then used to estimate a spectral type for each candidate companion (and target star when unknown). We also estimated a photomeric distance and its uncertainty based on the $J$-band magnitude and the spectral type-magnitude relation of Hawley et al. (2002) when the information was not available in the literature. We required that the estimated distances of the target and can- 
TABLE 1

PHOTOMETRIC DATA

\begin{tabular}{|c|c|c|c|c|c|c|c|c|c|}
\hline Name & 2MASS Name & $\alpha^{\mathrm{a}}$ & $\delta^{\mathrm{a}}$ & $r^{\prime \mathrm{b}}$ & $i^{\prime \mathrm{b}}$ & $z^{\prime \mathrm{b}}$ & $J^{\mathrm{a}}$ & $H^{\mathrm{a}}$ & $K_{s}^{\mathrm{a}}$ \\
\hline NLTT $251^{\mathrm{C}}$ & $\begin{array}{l}\text { MASS J00064746-0852350 } \\
\text { MASS J00064916-0852457 }\end{array}$ & $\begin{array}{l}00^{h} 06^{m} 47^{s} \cdot 47 \\
00^{h} 06^{m} 49^{s} .17\end{array}$ & $\begin{array}{lll}-08^{\circ} & 52^{\prime} & 35.1^{\prime \prime} \\
-08^{\circ} & 52^{\prime} & 45.7^{\prime \prime}\end{array}$ & $\begin{array}{l}17.30 \pm 0.01 \\
20.91 \pm 0.05\end{array}$ & $\begin{array}{l}4.94 \pm 0.01 \\
8.26 \pm 0.01\end{array}$ & $\begin{array}{l}13.73 \pm 0.01 \\
16.45 \pm 0.01\end{array}$ & $\begin{array}{l}11.97 \pm 0.02 \\
14.14 \pm 0.03\end{array}$ & $\begin{array}{l}11.43 \pm 0.02 \\
13.55 \pm 0.03\end{array}$ & $\begin{array}{l}11.09 \pm 0.02 \\
13.13 \pm 0.04\end{array}$ \\
\hline $\begin{array}{l}\text { NLTT } 687 \\
\end{array}$ & $\begin{array}{l}\text { IASS J00140212-1814578 } \\
\text { IASS J00135882-1816462 }\end{array}$ & $\begin{array}{l}00^{h} 14^{m} 02^{s} .10 \\
00^{h} 13^{m} 8^{s} .80 \\
\end{array}$ & $\begin{array}{lll}-18^{\circ} & 14^{\prime} & 58.0^{\prime \prime} \\
-18^{\circ} & 16^{\prime} & 46.2^{\prime \prime} \\
\end{array}$ & & & & $\begin{array}{l}10.38 \pm 0.02 \\
16.54 \pm 0.14\end{array}$ & $\begin{array}{l}9.81 \pm 0.02 \\
15.89 \pm 0.18\end{array}$ & $\begin{array}{c}9.57 \pm 0.02 \\
15.04 \pm 0.13\end{array}$ \\
\hline NLTT $2274^{\mathrm{d}}$ & $\begin{array}{l}\text { MASS J00415543+1341162 } \\
\text { MASS J00415453+1341351 }\end{array}$ & $\begin{array}{l}00^{h} 41^{m} m_{5}^{s} .43 \\
00^{h} 41^{m_{54}^{s}} .53\end{array}$ & 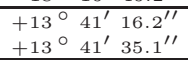 & $\begin{aligned} \cdots & g \\
21.15 & \pm 0.07\end{aligned}$ & $\begin{array}{l}5.42 \pm 0.01 \\
8.71 \pm 0.01\end{array}$ & $\begin{array}{c}\cdots \\
16.83 \pm 0.01\end{array}$ & $\begin{array}{l}10.16 \pm 0.03 \\
14.45 \pm 0.03\end{array}$ & $\begin{array}{c}9.57 \pm 0.03 \\
13.67 \pm 0.04\end{array}$ & $\begin{array}{c}9.35 \pm 0.02 \\
13.24 \pm 0.03\end{array}$ \\
\hline BD-06 813 & $\begin{array}{l}\text { MASS J04050271-0600261 } \\
\text { MASS J04050209-0600409 }\end{array}$ & $\begin{array}{l}4^{h} 05^{m} 02^{s} \cdot 71 \\
4^{h} 05^{m} 02^{s} \cdot 10\end{array}$ & $\begin{array}{lll}-06^{\circ} & 00^{\prime} & 26.1^{\prime \prime} \\
-06^{\circ} & 00^{\prime} & 40.9^{\prime \prime}\end{array}$ & $\begin{array}{ll} & \\
2 & \pm 0.06\end{array}$ & $\begin{array}{l}1.8 \\
\cdots 4 \pm 0.01\end{array}$ & $\begin{array}{l}\cdots \\
\cdots \\
08 \pm 0.01\end{array}$ & $\begin{array}{c}8.35 \pm 0.02 \\
15.11 \pm 0.05\end{array}$ & $\begin{array}{c}7.93 \pm 0.03 \\
14.30 \pm 0.05\end{array}$ & $\begin{array}{r}7.87 \pm 0.03 \\
13.88 \pm 0.05\end{array}$ \\
\hline NLTT $20640^{\mathrm{e}}$ & $\begin{array}{l}\text { 2MASS J08583671+2711068 } \\
\text { 2MASS J08583693+2710518 }\end{array}$ & $\begin{array}{l}08^{h} 58^{m} 36^{s} .72 \\
08^{h} 58^{m} 36^{s} .94\end{array}$ & $\begin{array}{lll}+27^{\circ} & 11^{\prime} & 06.8^{\prime \prime} \\
+27^{\circ} & 10^{\prime} & 51.8^{\prime \prime}\end{array}$ & $\begin{array}{l}15.92 \pm 0.01 \\
22.05 \pm 0.11\end{array}$ & $\begin{array}{l}\quad 8 \\
0.02 \\
\end{array}$ & $\begin{array}{l}13.50 \pm 0.01 \\
17.64 \pm 0.02\end{array}$ & $\begin{array}{l}11.99 \pm 0.02 \\
15.05 \pm 0.05\end{array}$ & $\begin{array}{l}11.40 \pm 0.02 \\
14.23 \pm 0.05\end{array}$ & $\begin{array}{l}11.10 \pm 0.01 \\
13.66 \pm 0.05\end{array}$ \\
\hline LSPM J1021+37 & $\begin{array}{l}2 \text { MASS J10215240+3704289 } \\
\text { 2MASS J10215386+3704166 }\end{array}$ & $\begin{array}{l}10^{h} 21^{m} 52^{s} \cdot 40 \\
10^{h} 21^{m_{53}} \cdot 87\end{array}$ & $\begin{array}{llll}+37^{\circ} & 04^{\prime} & 29.0^{\prime \prime} \\
+37^{\circ} & 04^{\prime} & 16.7^{\prime \prime}\end{array}$ & $\begin{array}{l}16.07 \pm 0.01 \\
24.67 \pm 0.48\end{array}$ & $\begin{aligned} g \\
\\
\end{aligned}$ & $\begin{array}{l}13.87 \pm 0.01 \\
19.91 \pm 0.08\end{array}$ & $\begin{array}{l}12.43 \pm 0.02 \\
17.18 \pm 0.03^{\mathrm{f}}\end{array}$ & $\begin{array}{l}11.95 \pm 0.02 \\
16.25 \pm 0.22\end{array}$ & $\begin{array}{l}11.76 \pm 0.02 \\
15.66 \pm 0.23\end{array}$ \\
\hline & $\begin{array}{l}\text { 2MASS J10432398-1706024 } \\
\text { 2MASS J10432513-1706065 }\end{array}$ & $\begin{array}{l}10^{h} 43^{m} 23^{s} .99 \\
10^{h} 43^{m} 25^{s} .13\end{array}$ & $\begin{array}{llll}-17^{\circ} & 06^{\prime} & 02.4^{\prime \prime} \\
-17^{\circ} & 06^{\prime} & 06.6^{\prime \prime} \\
\end{array}$ & & & & & & $\begin{array}{l}10.60 \pm 0.03 \\
14.61 \pm 0.11\end{array}$ \\
\hline NLTT 26746 & $\begin{array}{l}\text { 2MASS J11150134+1606447 } \\
\text { 2MASS J11150150+1607026 }\end{array}$ & $\begin{array}{l}11^{h} 15^{m} 01^{s} .35 \\
11^{h} 15^{m} 01^{s} .50\end{array}$ & $\begin{array}{l}+16^{\circ} 06^{\prime} 44.7^{\prime \prime \prime} \\
+16^{\circ}: 07^{\prime} \\
02.7^{\prime \prime}\end{array}$ & $\begin{array}{l}\ldots g \\
94 \pm 0.37\end{array}$ & $\begin{array}{l}16.61 \pm 0.01 \\
20.71 \pm 0.07\end{array}$ & $\begin{array}{l}g \\
0.05\end{array}$ & $\begin{array}{l}11.14 \pm 0.02 \\
16.40 \pm 0.12\end{array}$ & $\begin{array}{l}10.56 \pm 0.02 \\
15.22 \pm 0.09\end{array}$ & $\begin{array}{l}10.32 \pm 0.02 \\
14.56 \pm 0.11\end{array}$ \\
\hline NLTT 29392 & $\begin{array}{l}\text { IASS J12 } \\
\text { IASS J12 }\end{array}$ & $\begin{array}{l}12^{h} 02^{m} 49^{s} .63 \\
12^{h} 02^{m} 50^{s} .09\end{array}$ & $\begin{array}{l}+42^{\circ} 04^{\prime} \quad 47.5^{\prime \prime} \\
+42^{\circ} 04^{\prime} \quad 53.2^{\prime \prime}\end{array}$ & $\begin{array}{l}18.21 \pm 0.01 \\
21.45 \pm 0.07\end{array}$ & \pm 0.01 & $\begin{array}{l}87 \pm 0.01 \\
96 \pm 0.01\end{array}$ & & & $\begin{array}{l}11.67 \pm 0.02 \\
13.26 \pm 0.03\end{array}$ \\
\hline-1001 & $\begin{array}{l}\text { 2MASS J12594217+1001407 } \\
2 \text { MASS J12594167+1001380 }\end{array}$ & $\begin{array}{l}12^{h} 59^{m} 42^{s} \cdot 17 \\
12^{h} 59^{m} 41^{s} .68\end{array}$ & $\begin{array}{l}+10^{\circ} \quad 01^{\prime} \quad 40.7^{\prime \prime} \\
+10^{\circ} 01^{\prime} \quad 38.06^{\prime \prime}\end{array}$ & $\begin{array}{l}17.76 \pm 0.01 \\
24.45 \pm 0.76\end{array}$ & $\begin{array}{l}5.63 \pm 0.00 \\
.58 \pm 0.13\end{array}$ & $\begin{array}{l}14.44 \pm 0.00 \\
19.60 \pm 0.10\end{array}$ & $\begin{array}{l}12.60 \pm 0.02 \\
16.72 \pm 0.19\end{array}$ & $\begin{array}{l}12.03 \pm 0.03 \\
15.53 \pm 0.14\end{array}$ & $\begin{array}{l}11.63 \pm 0.02 \\
14.89 \pm 0.15\end{array}$ \\
\hline NLTT 36369 & $\begin{array}{l}\text { 2MASS J14081918+3708294 } \\
\text { 2MASS J14081969+3708255 }\end{array}$ & $\begin{array}{l}14^{h} 08^{m} 19^{s} \cdot 19 \\
14^{h} 08^{m} 19^{s} \cdot 70\end{array}$ & 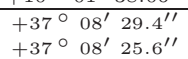 & $\begin{array}{l}15.60 \pm 0.01 \\
21.98 \pm 0.10\end{array}$ & $\begin{array}{l}4.53 \pm 0.01 \\
9.09 \pm 0.02\end{array}$ & $\begin{array}{l}13.48 \pm 0.01 \\
17.47 \pm 0.02\end{array}$ & $\begin{array}{l}12.06 \pm 0.02 \\
15.42 \pm 0.05\end{array}$ & $\begin{array}{l}11.45 \pm 0.02 \\
14.78 \pm 0.06\end{array}$ & $\begin{array}{l}11.22 \pm 0.02 \\
14.35 \pm 0.08\end{array}$ \\
\hline 66 & $\begin{array}{l}09+1856451 \\
65+1856484\end{array}$ & $\begin{array}{l}14^{h} 41^{m} 22^{s} .09 \\
14^{h} 41^{m} 25^{s} .65\end{array}$ & $\begin{array}{llll}+18^{\circ} & 56^{\prime} & 45.1^{\prime \prime} \\
+18^{\circ} & 56^{\prime} & 48.5^{\prime \prime}\end{array}$ & $\begin{array}{l}17.43 \pm 0.04 \\
23.38 \pm 0.27\end{array}$ & $\begin{array}{c}\cdots \\
21.34 \pm 0.07\end{array}$ & $\begin{array}{c}\cdots \\
19.41 \pm 0.06\end{array}$ & $\begin{array}{l}11.44 \pm 0.02 \\
16.94 \pm 0.18\end{array}$ & $\begin{array}{l}10.82 \pm 0.03 \\
16.17 \pm 0.17\end{array}$ & $\begin{array}{l}10.55 \pm 0.02 \\
15.40 \pm 0.13\end{array}$ \\
\hline NLTT 41701 & $\begin{array}{l}2 \text { MASS J } 15590815+3735477 \\
2 \text { MASS J } 15590740+3735275\end{array}$ & $\begin{array}{l}15^{h} 59^{m} 08^{s} \cdot 15 \\
15^{h} 59^{m} 07^{s} \cdot 40\end{array}$ & 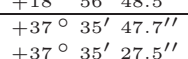 & $\begin{array}{c}23.38 \pm 0.27 \\
\cdots \cdot g \\
22.89+0.27\end{array}$ & 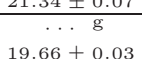 & 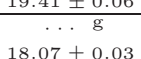 & $\begin{aligned} \frac{16.94 \pm 0.18}{9.12} \pm 0.02 \\
15.75 \pm 0.07\end{aligned}$ & $\begin{aligned} 16.17 \pm 0.17 \\
8.67 \pm 0.02 \\
14.92 \pm 0.07\end{aligned}$ & $\begin{array}{r}15.40 \pm 0.13 \\
8.56 \pm 0.02 \\
14.53+0.08\end{array}$ \\
\hline $\mathrm{HD}$ & $\begin{array}{l}\text { 2MASS J16460765+5020405 } \\
\text { 2MASS J16461148+5019456 }\end{array}$ & $\begin{array}{l}16^{h} 46^{m} 07^{s} .64 \\
16^{h} 46^{m} 11^{s} .48\end{array}$ & 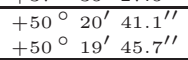 & $\begin{array}{l}\cdots g \\
\cdots 7 \pm 0.03\end{array}$ & $\begin{array}{ll} & \\
\cdots & g \\
43 & \pm 0.01\end{array}$ & $\begin{array}{l}\cdots \\
\cdots 6 \\
66.0 .01\end{array}$ & $\begin{array}{c}7.62 \pm 0.02 \\
13.61 \pm 0.02\end{array}$ & $\begin{array}{c}7.06 \pm 0.02 \\
12.98 \pm 0.03\end{array}$ & $\begin{array}{c}6.97 \pm 0.03 \\
12.59 \pm 0.02\end{array}$ \\
\hline HD 217246 & $\begin{array}{l}\text { 2MASS J22591790+0806484 } \\
\text { 2MASS J22591631+0806556 }\end{array}$ & $\begin{array}{l}22^{h} 59^{m} 17^{s} .90 \\
22^{h} 59^{m} 16^{s} .31\end{array}$ & 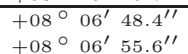 & $\begin{array}{c}\cdots g \\
21.83 \pm 0.09\end{array}$ & $\begin{array}{c}\cdots g \\
3.84 \pm 0.01\end{array}$ & $\begin{array}{c}\cdots g \\
17.61 \pm 0.02\end{array}$ & $\begin{array}{r}8.56 \pm 0.02 \\
15.76 \pm 0.06\end{array}$ & $\begin{array}{c}8.26 \pm 0.03 \\
15.14 \pm 0.08\end{array}$ & $\begin{array}{r}8.19 \pm 0.02 \\
14.74 \pm 0.10\end{array}$ \\
\hline NLTT & $\begin{array}{l}\mathrm{SS} \mathrm{J}_{23} \\
\mathrm{SS} \mathrm{J23}\end{array}$ & $\begin{array}{l}23^{h} 27^{m} 48^{s} \cdot 40 \\
23^{h} 27^{m} m_{49^{s}} \cdot 48\end{array}$ & $\begin{array}{l}+04^{\circ} 51^{\prime} 24.2^{\prime \prime \prime} \\
+04^{\circ} 50^{\prime} \quad 58.4^{\prime \prime}\end{array}$ & $\begin{array}{ll} & 8 \\
& 0.125\end{array}$ & $\begin{array}{ll} & 8 \\
\pm 0.02 & \end{array}$ & $\begin{array}{ll} & 8 \\
+0.02 & \end{array}$ & $\begin{array}{r}8.16 \pm 0.03 \\
15.10 \pm 0.03\end{array}$ & $\begin{array}{l}7.58 \pm 0.05 \\
14.36 \pm 0.04\end{array}$ & $\begin{array}{l}7.41 \pm 0.02 \\
13.97 \pm 0.05\end{array}$ \\
\hline TYC $1725-344-1$ & $\begin{array}{l}\text { 2MASS J23553113+1755239 } \\
\text { 2MASS J2355345+175404 }\end{array}$ & $\begin{array}{l}23^{h} 55 m 31^{s} \cdot 14 \\
23^{h} 55^{m} 34^{s} .50\end{array}$ & $\begin{array}{llll}+17^{\circ} & 55^{\prime} & 23.9^{\prime \prime} \\
+17^{\circ} & 54^{\prime} & 04.0^{\prime \prime}\end{array}$ & $\begin{array}{c}\cdots \mathrm{g} \\
23.24 \pm 0.33\end{array}$ & $\begin{array}{c}w^{\prime} \\
20.43 \pm 0.04\end{array}$ & $\begin{array}{c}\cdots \\
18.73 \pm 0.03\end{array}$ & $\begin{array}{c}8.80 \pm 0.03 \\
16.39 \pm 0.12\end{array}$ & $\begin{array}{c}8.38 \pm 0.02 \\
15.42 \pm 0.15\end{array}$ & $\begin{array}{c}8.28 \pm 0.02 \\
15.02 \pm 0.15\end{array}$ \\
\hline 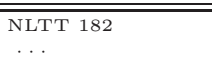 & $\begin{array}{l}\text { 2MASS J00054143+062625 } \\
\text { 2MASS J00054171+062630 }\end{array}$ & $\begin{array}{l}00^{h} 05^{m} 41^{s} .43 \\
00^{h} 05^{m} 41^{s} .71\end{array}$ & $\begin{array}{lll}+06^{\circ} & 26^{\prime} & 25.6^{\prime \prime} \\
+06^{\circ} & 26^{\prime} & 30.1^{\prime \prime}\end{array}$ & $\begin{array}{l}17.08 \pm 0.01 \\
22.38 \pm 0.16\end{array}$ & $\begin{array}{l}.42 \pm 0.04 \\
9.86 \pm 0.03\end{array}$ & $\begin{array}{l}14.51 \pm 0.01 \\
18.00 \pm 0.02\end{array}$ & $\begin{array}{l}13.02 \pm 0.03 \\
15.81 \pm 0.10\end{array}$ & $\begin{array}{l}12.51 \pm 0.03 \\
15.13 \pm 0.11\end{array}$ & $\begin{array}{l}12.19 \pm 0.03 \\
14.62 \pm 0.11\end{array}$ \\
\hline HD 2292 & $\begin{array}{l}2 \text { MASS J00265848+1705088 } \\
\text { 2MASS J0026599+170463 }\end{array}$ & 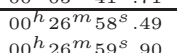 & $\begin{array}{lll}+17^{\circ} & 05^{\prime} & 0.8^{\prime \prime \prime} \\
+17^{\circ} & 04^{\prime} & 46.4^{\prime \prime}\end{array}$ & $\begin{array}{l}8.88 \pm 0.01 \\
21.82+0.13\end{array}$ & 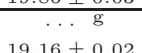 & $\begin{array}{c}\ldots g \\
\cdots 39+002\end{array}$ & $7.90 \pm 0.027$ & $7.55 \pm 0.04$ & $\begin{array}{l}7.50 \pm 0.03 \\
14.39+0.07\end{array}$ \\
\hline NLTT 4558 & $\begin{array}{l}\text { 2MASS J01221872+0330470 } \\
\text { 2MASS J01221697+0331235 }\end{array}$ & $\begin{array}{l}01^{h} 22^{m} 18^{s} .72 \\
01^{h} 22^{m} 16^{s} .98\end{array}$ & $\begin{array}{l}+03^{\circ} 30^{\prime}: 47.1^{\prime \prime} \\
+03^{\circ} 31^{\prime} 23.6^{\prime \prime}\end{array}$ & $\begin{array}{c}\cdots \\
22.05 \pm 0.13\end{array}$ & $\begin{array}{c}1.8 \\
.95 \pm 0.03\end{array}$ & $\begin{array}{c}\cdots \\
17.95 \pm 0.03\end{array}$ & $\begin{array}{c}7.42 \pm 0.02 \\
15.47 \pm 0.05\end{array}$ & $\begin{array}{c}7.14 \pm 0.03 \\
14.65 \pm 0.06\end{array}$ & $\begin{array}{c}7.07 \pm 0.02 \\
14.40 \pm 0.08\end{array}$ \\
\hline NLTT 30510 & $\begin{array}{l}\text { 2MASS J12221994+3643539 } \\
\text { 2MASS J12221837+3643485 }\end{array}$ & $\begin{array}{l}12^{h} 22^{m} 19^{s} .94 \\
12^{h} 2^{m} m_{18^{s}} \cdot 37\end{array}$ & 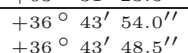 & $\begin{array}{l}38 \\
61 \pm 0.17\end{array}$ & $\begin{array}{l}38 \\
\cdots 1+0.03\end{array}$ & $\begin{array}{l}\cdots \\
\cdots \\
15 \\
150.03\end{array}$ & $\begin{array}{l}10.51 \pm 0.02 \\
15.97 \pm 0.08\end{array}$ & $\begin{array}{r}9.98 \pm 0.02 \\
15.27 \pm 0.09\end{array}$ & $\begin{array}{r}9.73 \pm 0.02 \\
14.85 \pm 0.09\end{array}$ \\
\hline LSPM & $\begin{array}{l}2 \text { MASS J123 } \\
\text { 2MASS J123 }\end{array}$ & $\begin{array}{l}12^{h} 36^{m} 35^{s} .59 \\
12^{h} 36^{m} 36^{s} .48\end{array}$ & $\begin{array}{llll}+30^{\circ} & 00^{\prime} & 31.6^{\prime \prime} \\
+30^{\circ} & 00^{\prime} & 31.5^{\prime \prime}\end{array}$ & $\begin{array}{l}19.63 \pm 0.02 \\
23.04 \pm 0.31\end{array}$ & $\begin{array}{l}17.75 \pm 0.01 \\
20.63 \pm 0.05\end{array}$ & $\begin{array}{l}16.81 \pm 0.01 \\
18.86 \pm 0.05\end{array}$ & $\begin{array}{l} \pm 0.05 \\
\pm 0.20\end{array}$ & $\begin{array}{l}0 \pm 0.06 \\
6 \pm 0.22\end{array}$ & $\begin{array}{l}4.30 \pm 0.08 \\
5.48 \pm 0.20\end{array}$ \\
\hline NLTT $33793^{\mathrm{d}}$ & $\begin{array}{l}\text { 2MASS J13205010+0955582 } \\
\text { 2MASS J13204159+0957506 }\end{array}$ & $\begin{array}{l}13^{h} 20^{m} 50^{s} .11 \\
13^{h} 20^{m} 41^{s} .59\end{array}$ & $\begin{array}{l}+09^{\circ} 55^{\prime} 58.3^{\prime \prime \prime} \\
+09^{\circ} 57^{\prime}: 50.6^{\prime \prime}\end{array}$ & $\begin{array}{c}\cdots g \\
20.40 \pm 0.03\end{array}$ & $\begin{array}{c}\cdots g \\
17.47 \pm 0.01\end{array}$ & $\begin{array}{c}\cdots g \\
15.80 \pm 0.01\end{array}$ & $\begin{array}{r}7.89 \pm 0.03 \\
13.73 \pm 0.03\end{array}$ & $\begin{array}{c}7.32 \pm 0.04 \\
13.08 \pm 0.03\end{array}$ & $\begin{array}{l}7.22 \pm 0.03 \\
12.61 \pm 0.03\end{array}$ \\
\hline
\end{tabular}

${ }^{a}$ Data are from the 2MASS catalog (Cutri et al.,2003)

${ }^{\mathrm{b}}$ Data are from the SDSS catalog Ahn et al. 2012

${ }^{c}$ This system has been identifified to be a hierarchical triple system with an M6 primary and an M9+T5 secondary by Burgasser et al. (2012)

d This system has been identifified as a binary by Faherty et al. (2010)

${ }^{\mathrm{e}}$ This system has been identifified as a binary by Zhang et all. (2010)

${ }^{\mathrm{f}}$ This magnitude has been extrated from a CPAPIR MKO J-band image and has been converted to the 2MASS magnitude system. This object was poorly detected in the 2MASS calatog as it is 
didate companion agree within the estimated uncertainties. Following this procedure, we identified 29 pairs of potential binary systems. We obtained imaging and spectroscopic follow-up observations of most of these candidates to improve their proper motion measurements and their spectral type determinations. We then applied a more detailed analysis, described below, to make a better assessment of their nature. Following this analysis, we found that 6 candidates systems are not comoving while 23 systems were found to be likely bound. These are the systems to be discussed in this paper (see Table 1).

Five of the 23 candidate pairs were found by other teams and reported as binary systems after we performed our initial search. The primary of these systems are NLTT 2274 (M4+M9.5), and NLTT 33793 (K4+M7.5), both found by Faherty et al. (2010), NLTT 20640 (Zhang et al. 2010, M4+L0), NLTT 251 (Burgasser et al. 2012, M7+M9+T5), and HD234344 (Mason et al. 2001a, $\mathrm{K} 7, \mathrm{M} 7)$. In addition, shortly prior to the submission of this manuscript, a new study by Deacon et al. (2014) reported the discovery of 4 other of our systems : NLTT 4558 (G5+L1+T3), NLTT56936 (K5+M8), NLTT26746 (M4+L4) and NLTT30510 (M2+M9.5).

\section{NEAR-INFRARED IMAGING}

\subsection{Observations}

We obtained $J$-band observations of 14 candidate companions between 2012 November and 2013 May using the Observatoire du Mont Mégantic (OMM) wide field near-infrared camera, CPAPIR Artigau et al. 2004), installed on the OMM $1.6 \mathrm{~m}$ telescope, and the OMM near-infrared spectroimager SIMON Albert 2006) installed on the CTIO $1.5 \mathrm{~m}$ telescope. The field of view (FOV) of CPAPIR at the OMM is $30^{\prime} \times 30^{\prime}$ with a pixel scale of $0.89^{\prime \prime}$ pixel $^{-1}$, while the FOV of SIMON at the Cerro Tololo Inter-American Observatory (CTIO) $1.5 \mathrm{~m}$ is $8.4^{\prime} \times 8.4^{\prime}$ with a pixel scale of $0.49^{\prime \prime}$ pixel $^{-1}$. Typically, the observations consisted of 15 individual exposures of 3 to $30 \mathrm{~s}$, with a dither step of $2^{\prime}$ between them. These observations provide a total time baseline of up to 15 years with respect to 2MASS for proper motion measurements.

\subsection{Data Reduction}

The reduction of the imaging data followed standard procedures. The images of SIMON and CPAPIR were sky subtracted using a median sky image constructed from the entire data set taken over the night in the $J$ filter. The images were flatfielded using a flat image derived from on and off dome images taken at the beginning or the end of the nights. Individual images astrometry was performed by cross-correlating point sources in the field with the 2MASS catalog. All images were then median-combined into a single science frame that was used for astrometric measurements.

\subsection{Results}

We measured the positions of the sources in our CPAPIR and SIMON follow-up imaging by finding all of the stars in the image and then fitting a 2D Gaussian over each one. These positions have been compared to those of all the objects in the 2MASS catalog that are up to 10' away from the target and the corresponding sources were associated. We determined the astrometric errors by computing the standard deviation of the difference between the positions in the CPAPIR/2MASS images and the corresponding positions in the 2MASS catalog propagated to the CPAPIR/SIMON epoch according to the proper motion listed in the NOMAD catalog (Zacharias et al. 2004); this was done for reference stars of brightness comparable to our targets. We determined the source positions in the SIMP data, also obtained with CPAPIR, in the same manner; those measurements are also given in Table 2 as they have not yet been published elsewhere.

For each candidate companion, we combined the position measurements from our follow-up imaging, 2MASS, SDSS, SIMP, and WISE to compute their proper motion using a weighted linear regression between the positions of the object and the measurement epochs. We adopted the uncertainty of the slope parameter as our uncertainty on the proper motion. The proper motions for all of our candidates, corrected for the parallax estimated from their photometric distance (see below), are compiled in Table 5. As an example, our calculated proper motions for all stars within $10^{\prime}$ of NLTT 26746 are plotted on Figure 1; the common proper motion of our candidate compan- 
TABLE 2

AstromETRIC MEASUREMENTS BASED ON OUR OBSERVATIONS

\begin{tabular}{|c|c|c|c|c|c|c|}
\hline Name & $\begin{array}{c}\text { RA } \\
(\operatorname{deg})\end{array}$ & $\begin{array}{c}\text { Uncertainty in RA } \\
\text { (mas) }\end{array}$ & $\begin{array}{l}\text { DEC } \\
(\operatorname{deg})\end{array}$ & $\begin{array}{c}\begin{array}{c}\text { Uncertainty in DEC } \\
\text { (mas) }\end{array} \\
\end{array}$ & $\begin{array}{c}\text { Epoch } \\
\text { (Reduced JD) }\end{array}$ & Source \\
\hline \multirow[t]{3}{*}{$2 \mathrm{M} 1043-1706 \mathrm{~A}$} & 160.84974 & 340 & -17.10118 & 400 & 54168.75 & SIMP \\
\hline & 160.84956 & 560 & -17.10128 & 670 & 56345.70 & SIMON \\
\hline & 160.84953 & 130 & -17.10125 & 130 & 56259.97 & CPAPIR \\
\hline \multirow[t]{3}{*}{$2 \mathrm{M} 1043-1706 \mathrm{~B}$} & 160.85452 & 340 & -17.10234 & 400 & 54168.75 & SIMP \\
\hline & 160.85435 & 220 & -17.10245 & 110 & 56345.70 & SIMON \\
\hline & 160.85433 & 250 & -17.10240 & 130 & 56259.97 & CPAPIR \\
\hline \multirow[t]{2}{*}{$2 \mathrm{M} 1646+5019$} & 251.54744 & 190 & 50.33065 & 190 & 55232.88 & SIMP \\
\hline & 251.54735 & 400 & 50.33103 & 180 & 56330.86 & CPAPIR \\
\hline \multirow[t]{2}{*}{ LSPM1259+1001 } & 194.92540 & 860 & 10.02808 & 720 & 55232.91 & SIMP \\
\hline & 194.92522 & 680 & 10.02804 & 270 & 56330.82 & CPAPIR \\
\hline \multirow[t]{3}{*}{$2 \mathrm{M} 1259+1001$} & 194.92335 & 860 & 10.02741 & 720 & 55232.91 & SIMP \\
\hline & 194.92339 & 320 & 10.02728 & 220 & 53900.55 & CPAPIR \\
\hline & 194.92327 & 380 & 10.02737 & 270 & 56330.82 & CPAPIR \\
\hline \multirow[t]{2}{*}{ LSPMJ1441+1856 } & 220.34193 & 380 & 18.94546 & 390 & 55232.92 & SIMP \\
\hline & 220.34189 & 160 & 18.94529 & 130 & 56330.89 & CPAPIR \\
\hline \multirow[t]{2}{*}{$2 \mathrm{M} 1441+1856$} & 220.35673 & 380 & 18.94627 & 390 & 55232.92 & SIMP \\
\hline & 220.37645 & 260 & 19.09413 & 290 & 56330.89 & CPAPIR \\
\hline NLTT182 & 1.42300 & 150 & 6.44028 & 170 & 53980.73 & SIMP \\
\hline \multirow{2}{*}{$2 \mathrm{M} 0005+0626$} & 1.42296 & 150 & 6.44028 & 170 & 53980.73 & SIMP \\
\hline & 1.43611 & 360 & 6.51388 & 410 & 56619.50 & SIMON \\
\hline \multirow[t]{2}{*}{ NLTT20640 } & 134.65331 & 310 & 27.18477 & 350 & 55144.94 & SIMP \\
\hline & 134.65344 & 120 & 27.18451 & 170 & 56329.48 & CPAPIR \\
\hline \multirow[t]{2}{*}{$2 \mathrm{M} 0858+2710$} & 134.65424 & 310 & 27.18052 & 350 & 55144.94 & SIMP \\
\hline & 134.67524 & 300 & 27.31993 & 340 & 56329.48 & CPAPIR \\
\hline \multirow[t]{2}{*}{ NLTT33793 } & 200.26834 & 190 & 10.02453 & 180 & 53838.67 & SIMP \\
\hline & 200.26833 & 360 & 10.02452 & 280 & 56330.83 & CPAPIR \\
\hline \multirow{2}{*}{$2 \mathrm{M} 1320+0957$} & 200.17290 & 190 & 9.96377 & 180 & 53838.67 & SIMP \\
\hline & 200.17247 & 110 & 9.96350 & 90 & 56330.83 & CPAPIR \\
\hline \multirow[t]{2}{*}{ NLTT36369 } & 212.07912 & 360 & 37.14177 & 250 & 55232.91 & SIMP \\
\hline & 212.08075 & 330 & 37.14089 & 870 & 56330.80 & CPAPIR \\
\hline \multirow[t]{2}{*}{$2 \mathrm{M} 1408+3708$} & 212.07911 & 360 & 37.14177 & 250 & 55232.91 & SIMP \\
\hline & 212.08084 & 380 & 37.14090 & 390 & 56330.80 & CPAPIR \\
\hline NLTT41701 & 239.78411 & 470 & 37.59615 & 400 & 55232.89 & SIMP \\
\hline & 239.78417 & 150 & 37.59600 & 160 & 56430.58 & CPAPIR \\
\hline $2 \mathrm{M} 1559+3735$ & 239.78101 & 470 & 37.59051 & 400 & 55232.89 & SIMP \\
\hline & 239.78105 & 120 & 37.59038 & 100 & 56430.58 & CPAPIR \\
\hline $2 \mathrm{M} 0122+0331$ & 20.57078 & 210 & 3.52294 & 150 & 54036.68 & SIMP \\
\hline NLTT56936 & 352.02213 & 130 & 4.97526 & 170 & 53986.70 & SIMP \\
\hline & 351.95329 & 290 & 4.85733 & 250 & 56572.54 & SIMON \\
\hline $2 \mathrm{M} 2327+0450$ & 351.95691 & 130 & 4.84984 & 170 & 53986.70 & SIMP \\
\hline & 351.95778 & 70 & 4.85024 & 290 & 56572.54 & SIMON \\
\hline $2 \mathrm{M} 0026+1704$ & 6.74936 & 220 & 17.07940 & 280 & 53994.73 & SIMP \\
\hline BD-06813 & 61.26145 & 470 & -6.00778 & 470 & 56349.54 & SIMON \\
\hline & 61.26139 & 140 & -6.00779 & 190 & 56258.78 & CPAPIR \\
\hline $2 \mathrm{M} 0405-0600$ & 61.25892 & 180 & -6.01190 & 180 & 56349.54 & SIMON \\
\hline & 61.25890 & 110 & -6.01187 & 110 & 56258.78 & CPAPIR \\
\hline NLTT2274 & 10.48038 & 360 & 13.68728 & 220 & 56619.54 & SIMON \\
\hline $2 \mathrm{M} 0041+1341$ & 10.47664 & 140 & 13.69254 & 70 & 56619.54 & SIMON \\
\hline $2 \mathrm{M} 2355+1754$ & 358.89345 & 360 & 17.90071 & 140 & 56619.54 & SIMON \\
\hline NLTT687 & 3.50881 & 1080 & -18.24962 & 2160 & 56547.70 & SIMON \\
\hline $2 \mathrm{M} 0013-1816$ & 3.49509 & 1080 & -18.27954 & 2160 & 56547.70 & SIMON \\
\hline $2 \mathrm{M} 2259+0806$ & 344.81841 & 140 & 8.11571 & 140 & 56570.54 & SIMON \\
\hline $2 \mathrm{M} 0006-0852$ & 1.70457 & 250 & -8.88072 & 220 & 56547.75 & SIMON \\
\hline LSPMJ1021+3704 & 155.46781 & 310 & 37.07414 & 70 & 56329.67 & CPAPIR \\
\hline $2 \mathrm{M} 1021+3704$ & 155.47389 & 490 & 37.07081 & 190 & 56329.67 & CPAPIR \\
\hline NLTT26746 & 168.75463 & 130 & 16.11187 & 90 & 56329.69 & CPAPIR \\
\hline $2 \mathrm{M} 1115+1607$ & 168.75524 & 220 & 16.11687 & 150 & 56329.69 & CPAPIR \\
\hline NLTT30510 & 185.58421 & 180 & 36.73147 & 110 & 56329.73 & CPAPIR \\
\hline $2 \mathrm{M} 1222+3643$ & 185.57769 & 290 & 36.72998 & 300 & 56329.73 & CPAPIR \\
\hline LSPMJ1236+3000 & 189.14888 & 120 & 30.00824 & 110 & 56329.71 & CPAPIR \\
\hline $2 \mathrm{M} 1236+3000$ & 189.15272 & 410 & 30.00832 & 210 & 56329.71 & CPAPIR \\
\hline $2 \mathrm{M} 1202+4204$ & 180.70753 & 580 & 42.08037 & 640 & 56330.73 & CPAPIR \\
\hline
\end{tabular}


ion with the primary is clearly seen.

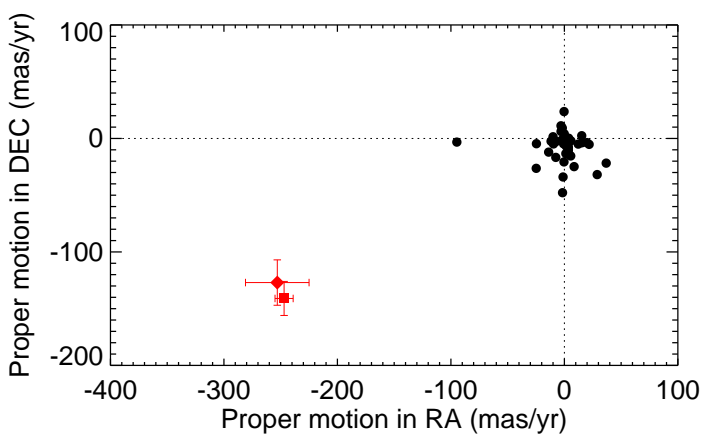

Fig. 1.- Proper motions for all objects within $10^{\prime}$ of NLTT 26746 based on our follow-up imaging (2MASS, SDSS, CPAPIR, and WISE data). The proper motion of the candidate companion $2 \mathrm{M} 1115+1607$ is shown with a red diamond, while the proper motion of the primary star is shown with a red square. The two components of the candidate binary have proper motions consistent within the estimated uncertainties.

\section{SPECTROSCOPY}

\subsection{Optical spectroscopy}

We obtained optical spectroscopy follow-up observations of all candidates using the Gemini Multi-Object Spectrographs (GMOS) Hook et al. 2004) at the Gemini South and North telescopes during semesters 2008A and 2008B (programs GN-2008A-Q-80, GN-2008A-Q-20, GS-2008A-Q12, GS-2008B-Q-34, and GN-2008B-Q-107). We used a $1^{\prime \prime}$ wide slit, the R400 grating with OG515 blocking filter, and 2-pixel binning in both the spatial and the spectral direction. For each target, we obtained exposures with three central wavelength settings, $790 \mathrm{~nm}, 800 \mathrm{~nm}$ and $810 \mathrm{~nm}$, to cover the small gaps between the three GMOS detectors. The exposure times range from 10 s to 600s (see Table 3). We did not move the target in the slit during the observations. The resulting spectra have a resolving power of $R \sim 850$ and cover the wavelength range from $650 \mathrm{~nm}$ to $1000 \mathrm{~nm}$. The typical seeing during the observations was $0.75^{\prime \prime}-1.0^{\prime \prime}$. Standard calibrations were obtained for each science data set. We used the calibration data of the G subdwarf LTT9239, the DA white dwarf EG 274 and the B0 star HZ44 for telluric and instrumental transmission calibration. Our main goal was to characterize the candidate companions, but given the short additional time needed, we also acquired a spectrum of the primary star during a visit to a given system. When the primary was not too bright to saturate the detector in an exposure of a few hundred seconds, both the primary and the companion were put in the slit simultaneously, otherwise a shorter observation of the primary was obtained after observation of the companion. Due to various issues during the execution of the observations, we were unable to procure data for all of the systems. In all, we obtained a spectrum for 14 candidate companions out for 23 and 10 candidate primaries out 23. A log of the observations is given in Table 3 .

The GMOS spectroscopy data were reduced using a custom Interactive Data Language (IDL) routine. First, we corrected the individual exposures for the bias and flat field. We then extracted the spectral trace using a Moffat function extraction profile, allowing for a linear sky solution. We subsequently constructed a quadratic wavelength solution using calibration arc lamps, and corrected the spectra for wavelength-dependent instrumental transmission using a standard spectrum. The reference spectroscopic spectra were taken from Hamuy et al. (1994) and Massey et al. (1988). In the last step, we combined the individual spectra extracted from each of the 3 detector section, and finally combined all exposures together for a given object. The measurement uncertainties were estimated by extracting an empty sky region near the spectral trace, using the same method. All these spectra are normalized at $0.75 \mu \mathrm{m}$. Figure 2 shows the GMOS spectra for 6 candidate primaries for which the spectral types were not published in the literature before this study. GMOS spectra at $R$ $\sim 850$ of ten candidates are shown in Figure 3 and the remaining four are shown in Figure 4 with $R \sim$ 400. The latter figure present the smoothed spectra our four faintest targets observed with GMOS. In all cases, a residual signal caused by an fringing effect from the detector is present. However, this effect affects the quality of our data significantly only for our faintest targets but even with this fringing, we are still able to identify the spectral type of the objects. 


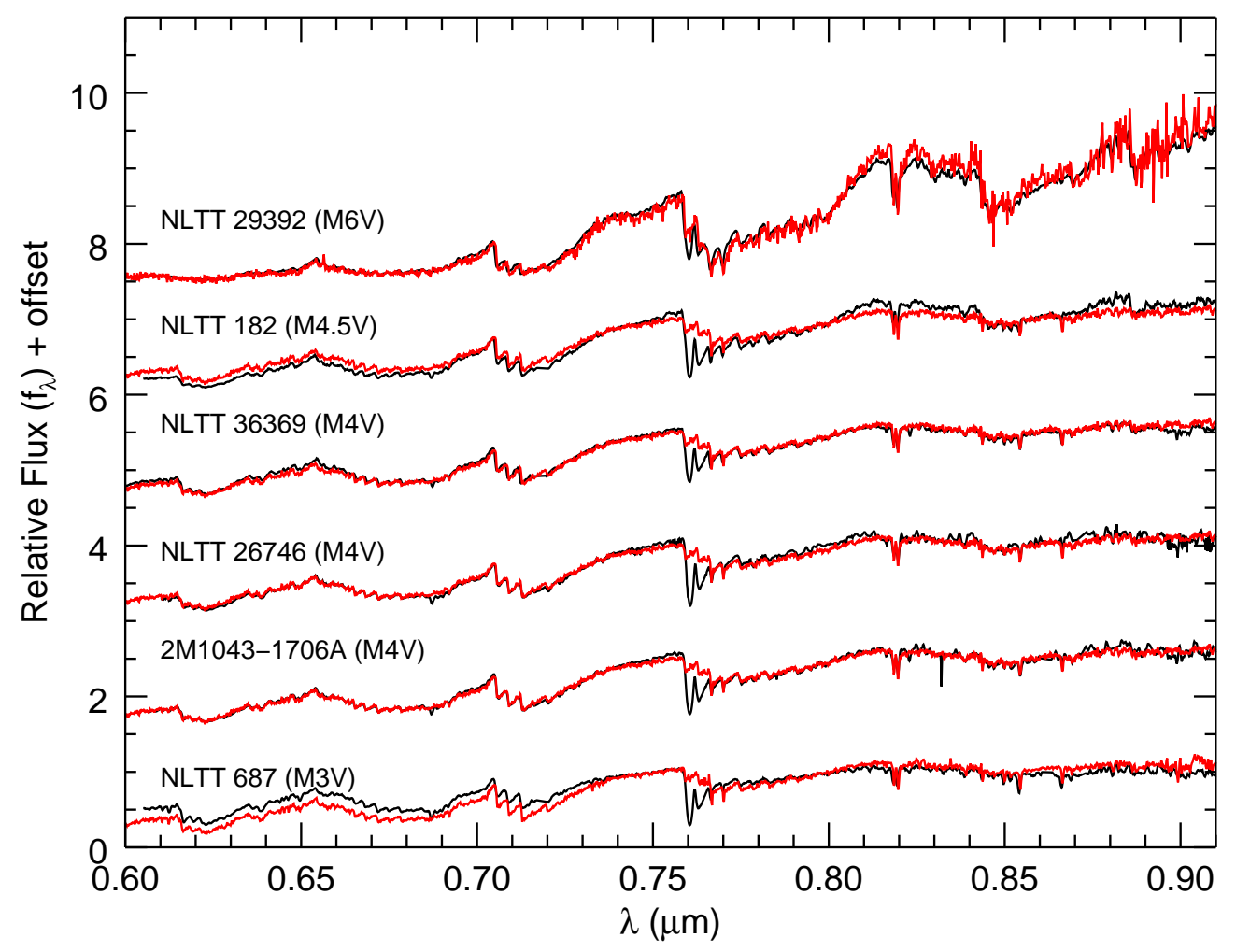

Fig. 2.- GMOS spectra of our 6 candidate primaries for which a spectral type was not available in the literature, displayed with a resolution of $\mathrm{R} \sim 850$. The spectra are normalized at $0.75 \mu \mathrm{m}$. As a comparison, we display in red the SDSS template spectra from Hawley et al. (2002) for the appropriate spectral type convolved to the same resolution. The feature in our spectra at $0.76 \mu \mathrm{m}$ is telluric absorption that we did not correct. 


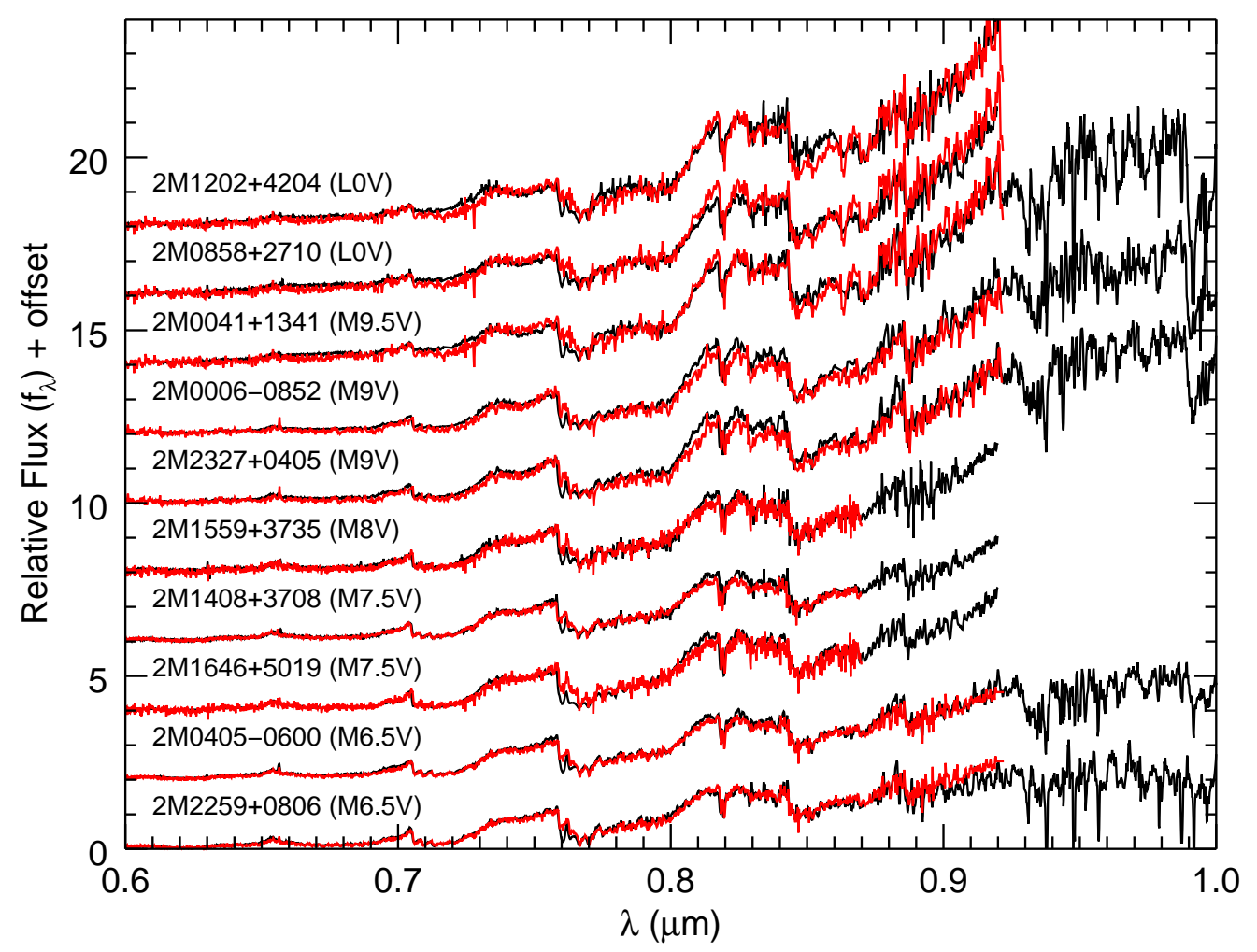

Fig. 3.- GMOS spectra of 10 of our candidate companions, displayed with a resolution of $\mathrm{R} \sim 850$. The spectra are normalized at $0.75 \mu \mathrm{m}$. As a comparison, we display in red the SDSS template spectra from Hawley et al. (2002) for the appropriate spectral type convolved to the same resolution. The feature in our spectra at $0.76 \mu \mathrm{m}$ is telluric absorption that we did not correct. Different observation setups account for the different wavelength range. 


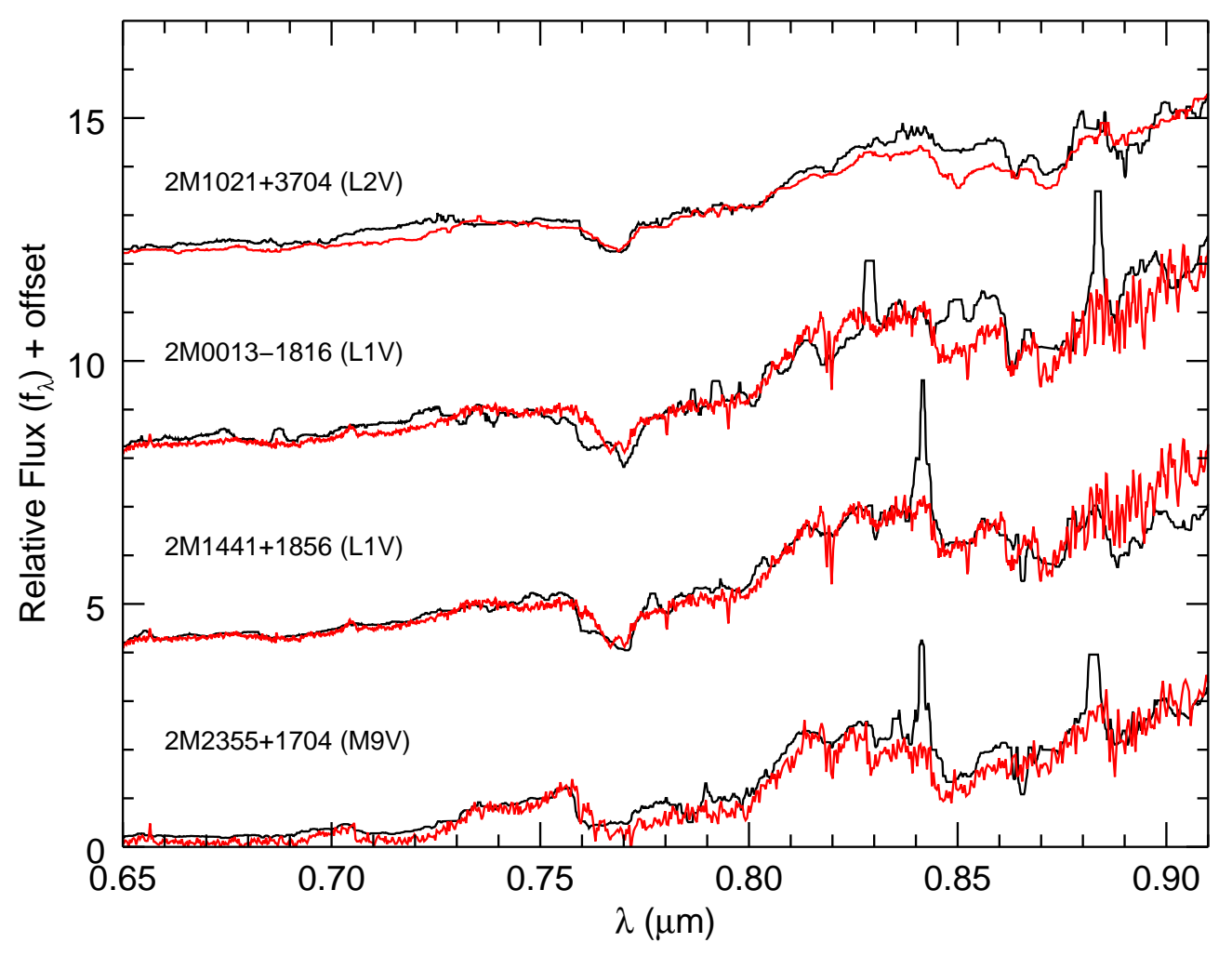

Fig. 4.- GMOS spectra of 4 of our candidate companions, displayed with a degraded resolution of $\mathrm{R} \sim$ 400 as these candidates were fainter and a lower signal-to-noise ratio was reached at full resolution. There is fringing in the data leading to a significant residual signal for these faint targets. The spectra are normalized at $0.75 \mu \mathrm{m}$. As a comparison, we display in red the SDSS template spectra from Hawley et al. (2002) for the appropriate spectral type. 


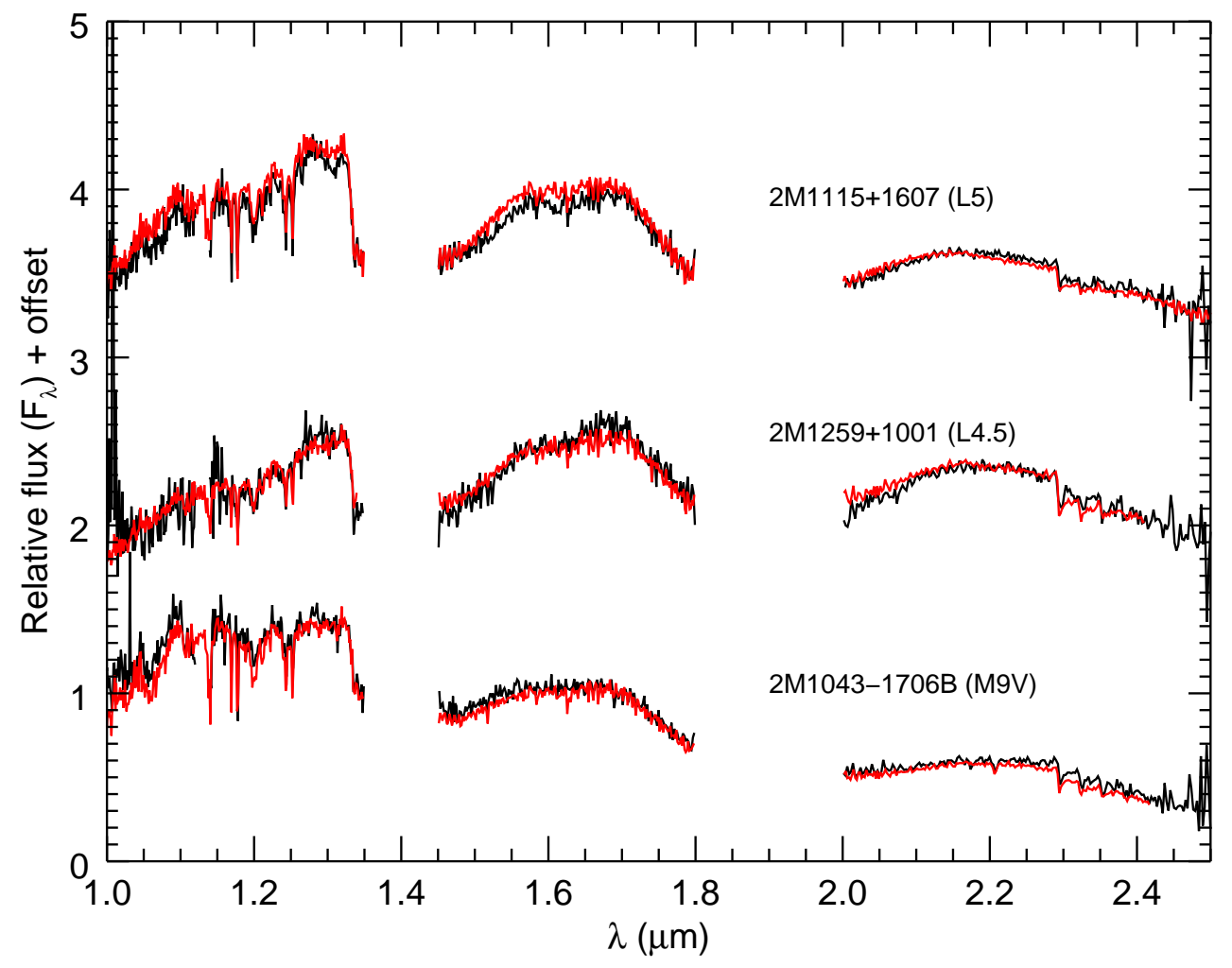

Fig. 5.- GNIRS spectra of 3 of our candidate companions, displayed with a spectral resolution of R 1700. The spectra are normalized at $1.1 \mu \mathrm{m}$. As a comparison, we display in red the spectra from SPEX IRTF Spectral Library (Cushing et al. 2005; Ravner et al. 2009) for the appropriate spectral type. 
TABLE 3

GMOS And GNIRS Spectroscopic Observations

\begin{tabular}{|c|c|c|c|c|c|c|}
\hline System & Component & $\begin{array}{c}\text { Obs. Date } \\
\text { YYYY-MM-DD }\end{array}$ & Instrument & Number of exposures & $\begin{array}{c}\text { Exp. time } \\
\text { (s) }\end{array}$ & Standard \\
\hline NLTT251 & Both & 2008-06-14 & GMOS-S & 6 & 600. & EG 274 \\
\hline \multirow[t]{2}{*}{ NLTT 687} & Primary & $2008-06-15$ & GMOS-S & 6 & 60.4 & EG 274 \\
\hline & Companion & $2008-06-15$ & GMOS-S & 6 & 600. & EG 274 \\
\hline \multirow{2}{*}{ NLTT 2274} & Primary & $2008-08-05$ & GMOS-S & 6 & 60.4 & LTT 9239 \\
\hline & Companion & 2008-08-05 & GMOS-S & 3 & 900. & LTT 9239 \\
\hline \multirow[t]{2}{*}{ BD-06 813} & Primary & $2008-01-28$ & GMOS-S & 6 & 60.4 & EG 274 \\
\hline & Companion & $2008-01-28$ & GMOS-S & 6 & 600. & EG 274 \\
\hline NLTT 20640 & Both & $2008-02-11$ & GMOS-N & 6 & 599. & $\mathrm{HZ} 44$ \\
\hline LSPM J1021+3704 & Both & $2008-02-27$ & GMOS-N & 2 & 599. & HZ44 \\
\hline $2 \mathrm{M} 1043-1706$ & Primary & $2008-01-28$ & GMOS-S & 6 & 600 . & HZ44 \\
\hline NLTT 26746 & Primary & $2009-01-01$ & GMOS-S & 3 & 900. & LTT 9239 \\
\hline NLTT 29392 & Both & $2008-02-11$ & GMOS-N & 6 & 599. & $\mathrm{HZ} 44$ \\
\hline NLTT 36369 & Both & $2008-04-14$ & GMOS-N & 9 & 599 . & HZ44 \\
\hline LSPM J1441+1856 & Companion & $2007-08-06$ & GMOS-S & 10 & 600 . & LTT 9239 \\
\hline \multirow{2}{*}{ NLTT 41701} & Primary & $2008-03-15$ & GMOS-N & $\frac{6}{6}$ & 9.99 & HZ44 \\
\hline & Companion & 2008-03-15 & GMOS-N & 9 & 599. & $\mathrm{HZ} 44$ \\
\hline \multirow[t]{2}{*}{ HD 234344} & Primary & $2008-03-02$ & GMOS-N & 6 & 29.9 & HZ44 \\
\hline & Companion & 2008-03-02 & GMOS-N & 9 & 419. & $\mathrm{HZ} 44$ \\
\hline HD 217246 & Companion & $2007-06-24$ & GMOS-S & 6 & 600. & LTT9239 \\
\hline NLTT 56936 & Companion & $2008-12-27$ & GMOS-S & 3 & 900. & LTT9239 \\
\hline TYC $1725-344-1$ & Companion & $2007-06-21$ & GMOS-S & 6 & 600 & LTT9239 \\
\hline NLTT 182 & Primary & $2008-06-30$ & GMOS-S & 6 & 600. & EG 274 \\
\hline $2 \mathrm{M} 1043-1706$ & Companion & $2007-04-01$ & GNIRS & 8 & 120. & HD 137873 \\
\hline $2 \mathrm{M} 1115+1607$ & Companion & $2007-01-08$ & GNIRS & 11 & 60.0 & HD 137873 \\
\hline $2 \mathrm{M} 1259+1001$ & Companion & $2007-01-08$ & GNIRS & $\frac{11}{15}$ & 60.0 & HD 137873 \\
\hline
\end{tabular}

\subsection{Near-Infrared Spectroscopy}

Three of our candidate companions, 2M10431706B, 2M1115+1607, and 2M1259+1001, were identified on their own as brown dwarf candidates in a separate search for high proper motion brown dwarfs in the SIMP data Artigau et al. 2009, Robert et al., in preparation), but have not yet been published. As other candidates identified in that search for brown dwarfs, they were followed-up using near-infrared spectroscopy with GNIRS at the Gemini South telescope in semester 2007A (GS-2007A-Q-28). We used a 0.3" wide slit, the $32 \mathrm{l} \mathrm{mm}^{-1}$ grating, the short camera, and the cross-dispersed mode for a resolving power of $R \sim 1700$ and a spectral coverage from $0.9 \mu \mathrm{m}$ to $2.5 \mu \mathrm{m}$. Observatory standard calibrations were obtained with each observation. For telluric and instrumental transmission calibration, the A0 star HD 137873 was observed shortly before/after each observation. The observations were obtained with a typical ABBA dither pattern along the slit with individual exposures of 60 to 120 s with 8 to 15 exposures. The details of the observations are shown in Table 3 .

The reduction of the spectra was made using custom $(I D L)$ routines as described in Delorme et al. (2008). First, successive image pairs were subtracted to remove the sky emission and divided by a median combined spectral flat. They were then corrected for both spectral and spatial distortions. To extract the spectra, a positive and a negative extraction box that matched the trace profile was used. An argon arc lamp spectrum was taken at the end of each sequence, which was used as a first wavelength calibration. The wavelength scale was then more precisely adjusted to the atmospheric $\mathrm{OH}$ lines (Rousselot et al. 2000). Finally, the spectra for each target extracted from image pairs were normalized and their median was taken to make the final spectra. The GNIRS spectra of the three companions are shown on Figure 5 along with a spectra from the SPEX IRTF Spectral Library Cushing et al. 2005; Ravner et al. 2009) 2 corresponding to the same spectral type as determined below.

\subsection{Results}

We determined the spectral type of all objects observed with GMOS from spectral indices, and then confirmed them by visual comparison with templates. For each spectrum we computed the following spectral indices: PC3, PC4, and PC5 as defined by Martin et al. (1996) and Martín et al. (1999), TiO5 and VO from Cruz \& Reid (2002), CaH, Ti I, and Ca II from Kirkpatrick et al. (1991), and VO 2 and TiO7 de-

\footnotetext{
${ }^{2}$ http://irtfweb.ifa.hawaii.edu/ spex/ IRTF_Spectral_Library
} 
fined by Lépine et al. (2003). Each of these indices constitutes a good spectral type indicator in some restricted spectral type interval, but altogether they cover the range from K5 to L6 dwarfs. The index values-spectral type relations and regimes of validity were taken from their respective papers. The measured indices are compiled in Table 4 along with the corresponding spectral types. The latter are averages of the spectral types obtained from all the indices that are valid for a given type. The few blanks in the table are caused by the shorter spectral coverage of some spectroscopic observations. We visually compared our spectra to templates from the Pickles Atlas (Pickles 1998) or to SDSS spectra of M and L dwarfs from Hawley et al. (2002) to confirm spectral typing. The spectral types of all companions observed with GMOS range from M6.5 to L1.

We determined the spectral type of the three companions observed with GNIRS using the spectral indices defined by Allers \& Liu (2013), which are based on the $\mathrm{H}_{2} \mathrm{O}, \mathrm{H}_{2} \mathrm{O}-1, \mathrm{H}_{2} \mathrm{O}-2$, and $\mathrm{H}_{2} \mathrm{OD}$ indices and which are valid for $\mathrm{M} 5 \mathrm{~V}$ to $\mathrm{L} 7 \mathrm{~V}$ dwarfs. Then, we visually compared our spectra to the templates of M and L dwarfs from the SPEX IRTF Spectral Library. We determined a spectral type of M9.0 \pm 0.5 for $2 \mathrm{M} 1043-1706 \mathrm{~B}, \mathrm{~L} 5 \pm 1$ for $2 \mathrm{M} 1115+1607$, and $\mathrm{L} 4.5 \pm 0.5$ for $2 \mathrm{M} 1259+1001$; these are indicated in Table 5 .

There are 19 objects for which we do not have GMOS nor GNIRS observations. Among these are 10 primaries and 2 companions for which a spectral type was known from the literature and we adopted those values. For the remaining seven, we estimated a spectral type from their 2MASS and SDSS colors based on the color-spectral type relations of Sheppard \& Cushing (2009). The spectral types adopted for all objects are summarized in Table 5 This table is divided in the two parts: the top half is for systems for which we have a spectrum for the companions while the lower half is for systems for which we did not have a spectrum for the companions.

For each object we estimated a photometric distance using their 2MASS $J$-band magnitude and the $M_{J}$-spectral type relations from Hawlev et al. (2002) for late-K to M5 dwarfs, and from Dupuy \& Liu (2012) for later type objects, M6 to L5. These distance estimates are given in Table 5

\section{PROBABILITY OF RANDOM ALIGN- MENT}

After determining the proper motion, the spectral types and the distance for all components, we need to confirm that they form a bound pair. The probability of random alignment is the probability that from chance, in a search like ours, we would find a physically unrelated companion star having the same proper motion and photometric distance as the primary, within our uncertainties, and that is separated by less than the observed separation of our candidate. This is given by the probability of finding two stars of the relevant spectral types within a given separation of each other and at the same distance, times the probability of finding two stars of the relevant spectral types with the same proper motion, given that they are at a similar location on the sky.

For the former probability, as our search targeted a fixed set of potential primary stars, we need only calculate the probability of finding a companion star close to those primaries. All of our candidate companions have spectral types in the M6-L5 range, thus this is the relevant range for statistical calculations. From the spatial density of M6-M8 dwarfs of $2.2 \times 10^{-3} \mathrm{pc}^{-3}$ per $I$-band magnitude interval determined by Phan-Bao et al. (2003) and the spatial density of M8-L3.5 of 1.64× $10^{-3} \mathrm{pc}^{-3}$ per $J$-band magnitude interval determined by Phan-Bao et al. (2008), we calculated the overall spatial density of late-M dwarfs to early-L dwarfs (M6 to L3.5). We used this density to calculate the number of such stars in a spherical shell of radius equal to the distance from the Sun to a candidate and thickness given by our distance estimate uncertainty. Then, from this number we calculated, the average number of stars in a sky projected disk of radius equal to the separation of our candidate binary, assuming an isotropic distribution of stars. We multiplied this number by 25 000, the number of primary stars targeted by our search, and finally calculated the corresponding probability of detecting at least one object within this total search area.

For the latter probability, we used Monte Carlo calculations to determine the proper motion distribution of stars of a given spectral type and at the sky position of the candidate system based on the observed Galactic space velocity $(U V W)$ dis- 
TABLE 4

Spectral Indices

\begin{tabular}{|c|c|c|c|c|c|c|c|c|c|c|c|}
\hline Object & $\mathrm{PC}^{\mathrm{a}}$ & $\mathrm{PC} 4^{\mathrm{a}}$ & $\mathrm{PC}^{\mathrm{a}}$ & $\mathrm{TiO}^{\mathrm{b}}$ & $\mathrm{VO}^{\mathrm{b}}$ & $\mathrm{CaH}^{\mathrm{c}}$ & $\mathrm{Ti} \mathrm{I}^{\mathrm{C}}$ & $\mathrm{Ca} \mathrm{II}{ }^{\mathrm{c}}$ & $\mathrm{VO} 2^{\mathrm{d}}$ & $\mathrm{TiO}^{\mathrm{d}}$ & Spectral Type ${ }^{e}$ \\
\hline NLTT251 & 1.3695 & 1.6816 & 1.7827 & 0.19484 & 2.1168 & 1.5603 & 1.0704 & 1.0764 & 0.56026 & 0.65387 & $\mathrm{M} 6 \pm 0.5$ \\
\hline $2 \mathrm{M} 0006-0852$ & 2.3435 & 3.8938 & 4.8902 & 0.34909 & 2.3607 & 1.5009 & 1.0326 & 1.0677 & 0.32328 & 0.46659 & M9 \pm 0.5 \\
\hline NLTT687 & 1.0306 & 1.0248 & 0.93255 & 0.58126 & 2.0021 & 1.1914 & 1.0622 & 1.2380 & 0.89734 & 0.92856 & $\mathrm{M} 3 \pm 0.5$ \\
\hline $2 \mathrm{M} 0013-1816$ & 2.8484 & 6.2637 & 7.3279 & 0.96672 & 2.3180 & 1.4236 & 1.0617 & 1.1923 & 0.60007 & 0.95069 & $\mathrm{~L} 1 \pm 0.5$ \\
\hline NLTT 2274 & 1.1037 & 1.1221 & 1.0918 & 0.40346 & 1.9970 & 1.2715 & 1.0803 & 1.1374 & 0.77698 & 0.85842 & $\mathrm{M} 4 \pm 0.5$ \\
\hline $2 \mathrm{M} 0041+1341$ & 2.7069 & 4.6043 & 5.7158 & 0.68651 & 2.5128 & 1.3602 & 1.0093 & 1.0645 & 0.36967 & 0.62286 & M9士0.5 \\
\hline $2 \mathrm{M} 0405-0600$ & 1.6339 & 2.3019 & 2.4712 & 0.26199 & 2.2343 & 1.3667 & 1.1098 & 1.1253 & 0.42198 & 0.57595 & $\mathrm{M} 6 \pm 1$ \\
\hline NLTT20640 & 1.1217 & 1.2361 & $\cdots$ & 0.37900 & 2.0244 & 1.3055 & 1.0679 & 1.1134 & 0.72927 & 0.85573 & $\mathrm{M} 4 \pm 0.5$ \\
\hline $2 \mathrm{M} 0858+2710$ & 2.4959 & 4.7033 & & 0.64835 & 2.3871 & 1.2406 & 0.98143 & 1.0637 & 0.38629 & 0.65786 & $\mathrm{~L} 0 \pm 1$ \\
\hline LSPMJ1021+3704 & 1.0898 & 1.1628 & $\cdots$ & 0.38845 & 1.9939 & 1.2819 & 1.0796 & 1.1583 & 0.75780 & 0.88121 & $\mathrm{M} 4 \pm 0.5$ \\
\hline $2 \mathrm{M} 1021+3704$ & 2.7920 & 5.3320 & & 1.0516 & 0.46435 & 0.92451 & $\ldots$ & 1.0668 & 0.60661 & 0.66820 & L2 \\
\hline $2 \mathrm{M} 1043-1706 \mathrm{~A}$ & 1.0665 & 1.1986 & 1.1118 & 0.41786 & 1.9828 & 1.2421 & & 1.1573 & 0.77439 & 0.87779 & $\mathrm{M} 4 \pm 0.5$ \\
\hline NLTT26746 & 1.0896 & 1.0664 & 1.1495 & 0.44003 & 1.9733 & 1.2758 & 1.1094 & 1.1651 & 0.83645 & 0.85031 & $\mathrm{M} 4 \pm 0.5$ \\
\hline NLTT29392 & 1.3948 & 2.0186 & $\cdots$ & 0.21082 & 2.1644 & 1.4311 & 1.0827 & 1.0974 & 0.49018 & 0.65474 & $\mathrm{M} 6 \pm 0.5$ \\
\hline $2 \mathrm{M} 1202+4204$ & 2.7094 & 5.3242 & $\cdots$ & 0.66899 & 2.4729 & 1.3948 & 0.96756 & 1.0718 & 0.39638 & 0.70797 & $\mathrm{LO} \pm 0.5$ \\
\hline NLTT36369 & 1.0359 & 1.0841 & $\cdots$ & 0.42531 & 2.0007 & 1.2596 & 1.0741 & 1.1489 & 0.80273 & 0.89932 & M4 \pm 0.5 \\
\hline $2 \mathrm{M} 1408+3708$ & 1.6805 & 2.5229 & $\cdots$ & 0.22558 & 2.3573 & 1.4836 & 1.0556 & 1.1543 & 0.37181 & 0.57255 & M7 \pm 0.5 \\
\hline $2 \mathrm{M} 1441+1856$ & 2.7019 & 4.2794 & 2.7264 & 0.88903 & 2.2368 & 1.5198 & 0.75043 & 1.2276 & 0.50723 & 0.76976 & $\mathrm{~L} 1 \pm 1$ \\
\hline $2 \mathrm{M} 1559+3735$ & 1.8393 & 2.9506 & $\cdots$ & 0.27357 & 2.4630 & 1.3777 & 1.0318 & 1.1406 & 0.33012 & 0.55086 & $\mathrm{M} 8 \pm 0.5$ \\
\hline $2 \mathrm{M} 1646+5019$ & 2.0450 & 3.0980 & $\cdots$ & 0.17044 & 2.1751 & 1.8641 & 1.0559 & 1.1482 & 0.39253 & 0.54864 & $\mathrm{M} 7 \pm 0.5$ \\
\hline $2 \mathrm{M} 2259+0806$ & 1.5610 & 1.8795 & 1.6930 & 0.14590 & 2.1279 & 1.5757 & 1.0905 & 1.2289 & 0.50888 & 0.61669 & M6 \pm 0.5 \\
\hline $2 \mathrm{M} 2327+0405$ & 2.1572 & 3.2263 & 3.8603 & 0.27870 & 2.4432 & 1.4433 & 1.0444 & 1.0839 & 0.33743 & 0.49614 & M9 \pm 0.5 \\
\hline $2 \mathrm{M} 2355+1754$ & 2.2927 & 3.7816 & 2.9846 & 0.42313 & 2.4953 & 1.3452 & 1.0084 & 1.1327 & 0.40961 & 0.76501 & M9 \pm 0.5 \\
\hline NLTT182 & 1.1426 & 1.2647 & 1.1793 & 0.34680 & 2.0167 & 1.3121 & 1.0885 & 1.1310 & 0.69285 & 0.79268 & $\mathrm{M} 4.5 \pm .5$ \\
\hline
\end{tabular}

${ }^{\mathrm{a}}$ The spectral indice is from Martin et al. (1996)

$\mathrm{b}_{\text {The spectral indice is from Cruz \& Reid (2002) }}$

${ }^{c}$ The spectral indice is from Kirkpatrick et al. (1991)

$\mathrm{d}$ The spectral indice is from Lépine et al. (2003)

e The spectral types shown in the last column are the median of the spectral types computed using the spectral indices presented in the table.

tributions of stars in the solar neighborhood. The $U V W$ distributions were derived from the velocity dispersions given by Mikami \& Heck (1982) for F, G and K stars, by Bochanski et al. (2011) for M dwarfs, and by Schmidt et al. (2010) for L dwarfs. We then determined the probability that a star of the spectral class of the primary and a star of the spectral class of the secondary, at the sky position of the system, would have proper motions larger than 0.1 mas yr$^{-1}$ and consistent with each other within $2 \sigma$.

We finally multiplied the above two probabilities to get the probability of random alignment; the results are given in Table 5. For all of our candidate systems, this probability is less than $1.3 \times 10^{-3}$, indicating that they most likely form a physical pairs.

\section{DISCUSSION}

We estimated the mass of the companions in our systems based on evolution models; this requires first estimating an age for the systems. The tangential velocity of our systems, which is an indicator of the population to which an object belongs, has been compared to the Besançon Galac- tic model (Robin et al. 2003). All of our systems have tangential velocities consistent with being part of the thin disk. According to the Galactic model simulations, most of our targets have a $>80 \%$ probability to be younger than 3 to 5 Gyr. We also looked for sign of youth in our GNIRS spectra. We used the spectral indices defined by Allers \& Liu (2013), already used to compute the spectral types, which are also sensitive to surface gravity. According to these indices, none of the three companions have low surface gravity. We then looked for $\mathrm{H} \alpha$ emission, at $656.3 \mathrm{~nm}$, which is indicative of the stellar activity and moderately correlated with age (West et al. 2008). Visual inspection of the spectra and calculations of the $\mathrm{H} \alpha$ equivalent widths indicate that $2 \mathrm{M} 0405$ 0600 (M6.5), 2M1202+4204 (L0) and 2M1043B (M9) have equivalent widths higher than $1 \AA$, and are thus active. Schmidt et al. (2010) had already identified that NLTT 29392B was an active L0 dwarf. However, this does not mean that they are particularly young as $\mathrm{M}$ dwarfs can be active during a few Gyr (see discussion in West et al. (2008))

\footnotetext{
${ }^{3}$ See http://www.vlmbinaries.org/ for an up-to-date census of the known very low mass binaries, maintained by $\mathrm{N}$. Siegler, C. Gelino and A. Burgasser.
} 
TABLE 5

DERIVED PARAMETERS FOR THE BINARY SYSTEMS

\begin{tabular}{|c|c|c|c|c|c|c|c|c|c|}
\hline Name & Spectral type ${ }^{a}$ & $\begin{array}{c}\mu_{\alpha} \cos \delta \\
\text { (mas/yr) }\end{array}$ & $\begin{array}{c}\mu_{\delta} \\
\text { (mas/yr) }\end{array}$ & $\begin{array}{c}\text { Separation } \\
(\prime \prime)\end{array}$ & $\begin{array}{c}\text { Distance }^{\mathrm{b}} \\
(\mathrm{pc})\end{array}$ & Prob $^{c}$ & $\begin{array}{c}\text { P. sep. } \\
(\mathrm{AU})\end{array}$ & $\begin{array}{c}\text { Pos. angle } \\
\text { (deg) }\end{array}$ & $\begin{array}{c}\operatorname{Mass} \mathrm{d} \\
\left(M_{\odot}\right)\end{array}$ \\
\hline NLTT $251^{p}$ & $\mathrm{M} 6.0 \pm 0.5$ & $-70 \pm 9$ & $-311 \pm 6$ & $27.4 \pm 0.6$ & $25_{-11}^{+7}$ & $2.8 \times 10^{-5}$ & $850_{-152}^{+84}$ & $113 \pm 1$ & $0.102-0.133$ \\
\hline 2M0006-0852 & M9.0 \pm 0.5 & $-59 \pm 18$ & $-318 \pm 9$ & & $37_{-11}^{+5}$ & & & & $0.079-0.085$ \\
\hline NLTT 687 & M3.0 \pm 0.5 & $-44 \pm 12$ & $-173 \pm 5$ & $118.1 \pm 0.6$ & $42_{-16}^{+26}$ & $3.9 \times 10^{-4}$ & $7400_{-1250}^{+1160}$ & $203.1 \pm 0.3$ & $0.389-0.412$ \\
\hline 2M0013-1816 & $\mathrm{L} 1.0 \pm 0.5$ & $-20 \pm 41$ & $-212 \pm 37$ & & $83_{-27}^{+7}$ & & & & $0.072-0.078$ \\
\hline NLTT $2274^{\mathrm{f}}$ & $\mathrm{M} 4.0 \pm 0.5$ & $-165 \pm 56$ & $-155 \pm 2$ & $23.2 \pm 0.8$ & $23_{-9}^{+14}$ & $1.6 \times 10^{-4}$ & $725_{-253}^{+230}$ & $325 \pm 2$ & $0.207-0.272$ \\
\hline $2 \mathrm{M} 0041+1341$ & $\mathrm{M} 9.5 \pm 0.5$ & $-162 \pm 58$ & $-153 \pm 13$ & & $40_{-12}^{+6}$ & & & & $0.076-0.083$ \\
\hline BD-06 813 & $\mathrm{~K}^{\circ}$ & $31 \pm 8$ & $-139 \pm 5$ & $17.4 \pm 0.2$ & $68 \pm 10^{h}$ & $7.6 \times 10^{-4}$ & $1340_{-340}^{+102}$ & $211.6 \pm 0.5$ & $0.925-1.014$ \\
\hline 2M0405-0600 & $\mathrm{M} 6.5 \pm 1$ & $42 \pm 6$ & $-117 \pm 10$ & & $86_{-37}^{+2}$ & & & & $0.096-0.114$ \\
\hline NLTT $20640^{\mathrm{q}}$ & M4.0 \pm 0.5 & $103 \pm 5$ & $-179 \pm 5$ & $15.6 \pm 0.2$ & $54_{-20}^{+32}$ & $2.2 \times 10^{-5}$ & $\begin{array}{l}780_{-270}^{+263} \\
\end{array}$ & $168.9 \pm 0.6$ & $0.207-0.272$ \\
\hline $2 \mathrm{M} 0858+2710$ & $\mathrm{~L} 0 \pm 1$ & $104 \pm 7$ & $-182 \pm 6$ & & $48_{-14}^{+3}$ & & & & $0.074-0.081$ \\
\hline LSPM J1021+3704 & $\mathrm{M} 4.0 \pm 0.5^{\mathrm{h}}$ & $-132 \pm 9$ & $-141 \pm 4$ & $22.2 \pm 0.4$ & $88_{-25}^{+39}$ & $1.3 \times 10^{-3}$ & $3000_{-675}^{+650}$ & $125 \pm 1$ & $0.207-0.272$ \\
\hline $2 \mathrm{M} 1021+3704$ & $\mathrm{~L} 0 \pm 1$ & $-131 \pm 8$ & $-124 \pm 12$ & & $93_{-36}^{+13}$ & & & & $0.071-0.076$ \\
\hline $2 \mathrm{M} 1043-1706 \mathrm{~A}$ & $\mathrm{M} 4.0 \pm 0.5$ & $-97 \pm 16$ & $-140 \pm 22$ & $17.1 \pm 0.1$ & $42_{-16}^{+26}$ & $7.1 \times 10^{-4}$ & $1020_{-306}^{+288}$ & $84 \pm 0.5$ & $0.207-0.272$ \\
\hline $2 \mathrm{M} 1043-1706 \mathrm{~B}$ & M9.0 \pm 0.5 & $-90 \pm 14$ & $-141 \pm 22$ & & $75_{-23}^{+6}$ & & & & $0.079-0.085$ \\
\hline NLTT $26746^{\mathrm{u}}$ & M4.0 \pm 0.5 & $-247 \pm 8$ & $-141 \pm 2$ & $18.0 \pm 0.2$ & $36_{-14}^{+22}$ & $1.5 \times 10^{-4}$ & $660_{-441}^{+216}$ & $6.5 \pm 0.7$ & $0.207-0.272$ \\
\hline $2 \mathrm{M} 1115+1607$ & $\mathrm{~L} 5 \pm 1^{\mathrm{t}}$ & $-253 \pm 28$ & $-127 \pm 20$ & & $37_{-35}^{+3}$ & & & & $0.056-0.073$ \\
\hline NLTT 29392 & $\mathrm{M} 6.0 \pm 0.5$ & $-306 \pm 11$ & $-300 \pm 17$ & $7.3 \pm 0.1$ & $33_{-16}^{+2}$ & $1.1 \times 10^{-5}$ & $310_{-135}^{+16}$ & $42.0 \pm 0.8$ & $0.102-0.133$ \\
\hline $2 \mathrm{M} 1202+4204$ & $\mathrm{~L} 0.0 \pm 0.5$ & $-292 \pm 6$ & $-276 \pm 11$ & & $38_{-11}^{+2}$ & & & & $0.074-0.081$ \\
\hline LSPM J1259+1001 & $\mathrm{M} 5 \pm 1^{\mathrm{g}}$ & $-142 \pm 15$ & $22 \pm 9$ & $7.65 \pm 0.08$ & $42_{-21}^{+44}$ & $2.9 \times 10^{-3}$ & $345_{-156}^{+196}$ & $250.9 \pm 0.6$ & $0.121-0.167$ \\
\hline $2 \mathrm{M} 1259+1001$ & $\mathrm{~L} 4.5 \pm 0.5$ & $-146 \pm 5$ & $29 \pm 7$ & & $47_{-18}^{+5}$ & & & & $0.057-0.074$ \\
\hline NLTT 36369 & M4.0 \pm 0.5 & $-247 \pm 8$ & $80 \pm 1$ & $7.9 \pm 0.1$ & $55_{-21}^{+33}$ & $9.3 \times 10^{-5}$ & $590_{-180}^{+162}$ & $122 \pm 1$ & $0.207-0.272$ \\
\hline $2 \mathrm{M} 1408+3708$ & M $7.5 \pm 0.5$ & $-249 \pm 1$ & $80 \pm 10$ & & $84_{-29}^{+4}$ & & & & $0.087-0.096$ \\
\hline LSPM J1441+1856 & $\mathrm{M}^{\mathrm{h}}$ & $-56 \pm 9$ & $-154 \pm 9$ & $51.1 \pm 0.2$ & $56_{-10}^{+37}$ & $8.7 \times 10^{-4}$ & $4110_{-1166}^{+1219}$ & $86.4 \pm 0.2$ & $0.102-0.133$ \\
\hline $2 \mathrm{M} 1441+1856$ & $\mathrm{~L} 1 \pm 1$ & $-59 \pm 9$ & $-185 \pm 12$ & & $99_{-34}^{+10}$ & & & & $0.072-0.079$ \\
\hline NLTT 41701 & $\mathrm{~K} 2^{\mathrm{h}}$ & $42 \pm 12$ & $-137 \pm 9$ & $22.4 \pm 0.5$ & $\left(61_{-27}^{+61}\right)^{\mathrm{i}}$ & $8.1 \times 10^{-4}$ & $1735_{-644}^{+690}$ & $204 \pm 1$ & $0.872-0.941$ \\
\hline $2 \mathrm{M} 1559+3735$ & M $8.0 \pm 0.5$ & $46 \pm 9$ & $-152 \pm 14$ & & $89_{-29}^{+6}$ & & & & $0.084-0.090$ \\
\hline HD $234344^{\mathrm{S}}$ & $\mathrm{K} 7^{\mathrm{h}}$ & $-129 \pm 29$ & $394 \pm 11$ & $69.3 \pm 0.3$ & $31.6 \pm 0.9^{\mathrm{m}}$ & $2.4 \times 10^{-4}$ & $2700_{-593}^{+158}$ & $146.2 \pm 0.3$ & $0.705-0.751$ \\
\hline $2 \mathrm{M} 1646+5019$ & $\mathrm{M} 7.5 \pm 0.5$ & $-118 \pm 4$ & $400 \pm 7$ & & $36_{-14}^{+3}$ & & & & $0.087-0.095$ \\
\hline HD217246 & $\mathrm{G} 5^{\mathrm{h}}$ & $137 \pm 34$ & $59 \pm 19$ & $24.7 \pm 0.2$ & $\left(68_{-32}^{+70}\right)^{\mathrm{i}}$ & $3.6 \times 10^{-4}$ & $2340_{-1000}^{+1125}$ & $286.0 \pm 0.5$ & $0.821-0.930$ \\
\hline $2 \mathrm{M} 2259+0806$ & $\mathrm{M} 6.5 \pm 0.5$ & $122 \pm 6$ & $69 \pm 5$ & & $121_{-51}^{+20}$ & & & & $0.096-0.113$ \\
\hline NLTT $56936^{\mathrm{u}}$ & $\mathrm{K} 2+\mathrm{K} 5^{\mathrm{n}}$ & $443 \pm 1$ & $171 \pm 8$ & $30.3 \pm 0.1$ & $64 \pm 9^{k}$ & $4.7 \times 10^{-5}$ & $1825_{-390}^{+180}$ & $147.8 \pm 0.2$ & $1.46 \pm 0.09^{\mathrm{n}}$ \\
\hline $2 \mathrm{M} 2327+04505$ & M9 \pm 0.5 & $443 \pm 6$ & $191 \pm 12$ & & $57_{-17}^{+3}$ & & & & $0.079-0.085$ \\
\hline TYC $1725-344-1$ & G5III $^{h}$ & $-65 \pm 21$ & $-78 \pm 14$ & $93.8 \pm 0.4$ & $\left(40_{-15}^{+28}\right)^{\mathrm{i}}$ & $2.8 \times 10^{-4}$ & $6700_{-2470}^{+1805}$ & $149.0 \pm 0.23$ & $1.91 \pm 0.11^{\mathrm{h}}$ \\
\hline $2 \mathrm{M} 2355+1754$ & M9.0 \pm 0.5 & $-69 \pm 16$ & $-86 \pm 3$ & & $103_{-37}^{+10}$ & & & & $0.079-0.085$ \\
\hline NLTT 182 & $\overline{\mathrm{M} 4.5 \pm 0.5}$ & $\overline{213 \pm 6}$ & $-113 \pm 7$ & $\overline{6.1 \pm 0.1}$ & $762_{-23}^{+38}$ & $\overline{1.4 \times 10^{-4}}$ & $\begin{array}{l}400_{-135}^{+126} \\
\end{array}$ & $\overline{43 \pm 4}$ & $0.157-0.181$ \\
\hline $2 \mathrm{M} 0005+0626$ & $\mathrm{~L} 0 \pm 1^{\mathrm{g}}$ & $205 \pm 7$ & $-126 \pm 8$ & & $69_{-22}^{+5}$ & & & & $0.079-0.085$ \\
\hline LSPM J1236+3000 & $\mathrm{M} 6 \pm 1^{\mathrm{g}}$ & $140 \pm 5$ & $-124 \pm 4$ & $12.2 \pm 0.8$ & $112_{-52}^{+6}$ & $1.9 \times 10^{-3}$ & $1580_{-624}^{+156}$ & $89 \pm 4$ & $0.102-0.133$ \\
\hline $2 \mathrm{M} 1236+3000$ & $\mathrm{M} 9.0 \pm 1^{\mathrm{g}}$ & $162 \pm 27$ & $-102 \pm 15$ & & $126_{-44}^{+18}$ & & & & $0.079-0.085$ \\
\hline HD 2292 & G5 ${ }^{r}$ & $-143 \pm 15$ & $-113 \pm 19$ & $30.5 \pm 0.5$ & $\left(42_{-14}^{+28}\right)^{\mathrm{i}}$ & $2.6 \times 10^{-4}$ & $1200_{-510}^{+180}$ & $138 \pm 1$ & $1.05-1.12$ \\
\hline $2 \mathrm{M} 0026+1704$ & $\mathrm{M} 9 \pm 1 \mathrm{~g}$ & $-149 \pm 2$ & $-128 \pm 41$ & & $65_{-20}^{+4}$ & & & & $0.108-0.118$ \\
\hline NLTT $30510^{\mathrm{u}}$ & $\mathrm{M} 3 \pm 1^{\mathrm{g}}$ & $269 \pm 12$ & $-39 \pm 16$ & $20.7 \pm 0.5$ & $45_{-23}^{+47}$ & $1.9 \times 10^{-4}$ & $1635_{-384}^{+648}$ & $254.2 \pm 0.9$ & $0.377-0.431$ \\
\hline $2 \mathrm{M} 1222+3643$ & $\mathrm{LO}^{\mathrm{e}}$ & $272 \pm 18$ & $-36 \pm 8$ & & $70 \pm 10^{1}$ & & & & $0.074-0.081$ \\
\hline NLTT $33793^{j}$ & $\mathrm{~K} 5^{\mathrm{j}}$ & $-210 \pm 64$ & $-131 \pm 12$ & $168.5 \pm 0.3$ & $\left(38.1_{-2.3}^{+2.6}\right)^{j}$ & $5.4 \times 10^{-5}$ & $5780_{-1190}^{+2040}$ & $311.7 \pm 0.1$ & $0.724-0.782$ \\
\hline $2 \mathrm{M} 1320+0957$ & $\mathrm{M}_{8}^{\mathrm{j}}$ & $-222 \pm 38$ & $-141 \pm 10$ & & $36 \pm 3^{j}$ & & & & $0.087-0.096$ \\
\hline NLTT $4558^{\mathrm{u}}$ & $\mathrm{G}^{\mathrm{m}}$ & $22 \pm 13$ & $-142 \pm 15$ & $44.8 \pm 0.8$ & $\left(58 \pm 3^{\mathrm{k}}\right.$ & $3.5 \times 10^{-5}$ & $2222_{-960}^{+308}$ & $324.2 \pm 1$ & $1.05-1.12$ \\
\hline $2 \mathrm{M} 0122+0331$ & $\mathrm{~L} 2 \pm 1 \mathrm{~g}$ & $45 \pm 10$ & $-162 \pm 5$ & & $43_{-40}^{+13}$ & & & & $0.071-0.076$ \\
\hline
\end{tabular}

${ }^{a}$ Spectral type are extracted from our GMOS or GNIRS spectra unless noted otherwise, ${ }^{b}$ Distance computed using the spectral types and the relations from Dupuy \& Liu (2012) and Hawley et al. (2002), ${ }^{c}$ Probability of random alignement, ${ }^{d}$ Masses are evaluated using the mass-M $J$ relation from the BT-Settl model of Allard (2014), EZhang et al. (2009a), ${ }^{f}$ This system has been identified as a M4+Lo binary by Faherty et al. (2010). Their spectral types, proper motions, distance and sepration are consistent with the ones we found, $g$ Spectral type calculated from i'-J (Sheppard \& Cushing [2009), ${ }^{h}$ [Pickles \& Depagne [2010), ${ }^{i}$ Ammons et al. 2006) has estimated a distance that matches to one obtained from the spectral type extracted from the optical GMOS spectrum, ${ }^{j}$ This system has been identified as a M4+LO binary by Faherty et a1. 2010), ${ }^{k}$ Heliocentric distance from Anderson \& Francis (2013), ${ }^{l}$ Photometric distance from [Zhang et al. (2009a]), $m$ Heliocentric distance from Anderson \& Francis (2013), ${ }^{n}$ Hrivnak et al. (1995), ${ }^{o}$ The spectral type is from Barney (1951), ${ }^{p}$ This system has been identified to be a hierarchical triple system with an M7.0 \pm 0.5 primary and an M $8.5 \pm 0.5+\mathrm{T} 5 \pm 1$ secondary by (Burgasser et al. (2012). Their proper motions, distance and sepration are consistent with the are conses proper motions, distance and sepration are consistent with the ones we found, ${ }^{t}$ Zhang et al. (2009b) found a spectral type of L1 by colors and has identified it as a
brown dwarf candidate., ${ }^{u}$ This system has been identified by (Deacon et al. (2014), ${ }^{v}$ The mass of NLTT 4558 has been calculated by (Porto de Mello et al. (2014) 


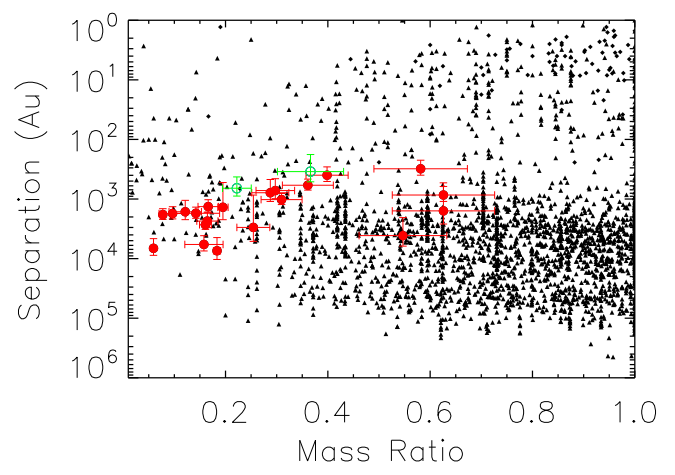

Fig. 6.- Separation (in AU) versus mass ratio for our systems and for known binaries. The red circles represent our sample. The LSPMJ1259+1001 system and the NLTT26746 system, for which the companion is either a L4.5 or a L5 dwarf, are shown with the open green circle. Very low mass binaries from the Very Low Mass Binaries Archiv 3 are shown as a diamond while the triangles represent binary systems from Gomes et al. (2013) and references therein, Janson et al. (2012), Dhital et al. (2010), Fahertv et al. (2010) and references therein, Raghavan et al. (2010), Metchev \& Hillenbrand (2004), Reid et al. (2001), Wilson et al. (2001) Mason et al. (2001b), Reid \& Gizis (1997), Fischer \& Marcy (1992), and Close et al. (1990).

on the relatively weak constraint that $\mathrm{H} \alpha$ provides for an individual object).

In addition, we used the BANYAN II code Gagné et al. 2014) to test if any of our targets belong to a nearby young moving group. We found out that while most of our target have a higher probability of belonging to the field, one of them (NLTT182) has a $98.8 \%$ probability of being a Beta Pictoris member, with a corresponding false positive rate of $4.5 \%$. This is quite interesting but by no means a proof of youth or true membership; the complete 3D kinematics would have to be confirmed, as well as youth from various indicators. We only have an optical spectrum of the primary star of this system, and we looked for gravity-sensitive spectroscopic indices in it (Kirkpatrick et al. 2000; Cruz \& Reid 2002; Cruz et al. 2007; Reid et al. 2008), such as the Naa index, the Na-b index, the K-a index, and the
CrH-a index, to assess whether this object is young or not. However, these four indices fall close to the field scatter and the result of our analysis is mainly inconclusive. More observations will be necessary to firmly establish whether or not this system is young. For the purpose of this paper, due to the lack of solid evidence, we simply assume this system to be of the age of the field, as we do for all of our other systems.

Based on the above analyses, we thus estimated that the ages of all of the systems are roughly 1-7 Gyr. We then evaluated the masses using the mass $-\mathrm{M}_{J}$ relation from the BT-Settl model of Allard (2014), where the $\mathrm{M}_{J}$ are obtained from our inferred spectral types using the relation of Hawlev et al. (2002) and Dupuy \& Liu (2012). In the cases where the distance were not well defined, we decided to use the effective temperature instead of the $J$ magnitude to find the masses. The effective temperature for each target has been extracted from its spectral type according to the relations in Pecaut \& Mamajek (2013). We note that masses infered in this manner are overestimated by about $10 \%$ for objects with a G or early-K spectral types as compared to Henry \& McCarthy (1993). The estimated masses for ages between 1 to 7 Gyr are given in Table 5 The uncertainties come from the propagation of the error of spectral types on the inferred magnitudes (or effective temperatures).

Figure [ shows the separation in AU of our systems as a function of their mass ratio, as compared with other known binaries. Some of our new systems, having low mass ratios, reach a sparsely populated regions of the diagram. In particular, we found 14 systems with a mass ratio less than 0.3 , below the bulk of the previously known population.

As some of our system have a low binding energy, it is interesting to find out if the systems are stable. Orbital evolution is possible for wide, weakly bound binaries and it might lead to their disruption as they travel through the Galaxy and encounter stars and giants molecular clouds. Weinberg et al. (1987) worked out the calculations for the evolution and lifetimes of such wide binary systems in the solar neighbourhood; these can easily be scaled to very low-mass stars as was done by Artigau et al. (2007). For all of our binaries, we find that the half-life is of the order of a Hubble 
time or larger. The probability of survival is thus high for all systems.

Burgasser et al. (2005) have shown that the binary fraction is higher for ultra cold dwarfs that are in a wide binary system where the primary is a stellar object. Furthermore, Whitworth \& Stamatellos (2006) discussed the possibility that $\mathrm{H}_{2}$ dissociation might trigger a secondary fragmentation of a companion low-mass protostar if the latter is at the cooler outer parts of the circumstellar disk ( $>100 \mathrm{AU})$ and is spinning at a high enough rate. Thus, it is possible that some of our companions (and perhaps primaries) are unresolved binaries themselves. If that were the case, this could be reconciled with our common photometric distance estimates given the large associated uncertainties and could be tested observationally with either adaptive optics observations or high-precision radial-velocity monitoring of the primary.

To differentiate the evolutionary states of our stars, we use the four spectral classes of metallicity defined by Lépine et al. (2007) that are defined by the $\zeta_{\mathrm{Ti05} / \mathrm{CaH}}$ index, which is based on the $\mathrm{CaH} 2$, CaH3 and TiO5 molecular bands in the optical. This index has been re-calibrated by Dhital et al. (2012) and is used to differentiate dwarfs, subdwarfs, extreme subdwarfs and ultrasubdwarfs. Using this index for all of our M dwarfs, we found that they all have $\zeta_{\mathrm{Ti05/CaH}}>0.85$, meaning that they all are in the dwarf metallicity class with near-solar metallicity.

\section{CONCLUSION}

We have discovered 14 new binary systems with companions of spectral types M6-L5 at separations of $6^{\prime \prime}-170^{\prime \prime}$ from their primaries, corresponding to projected separations of $250-7500 \mathrm{AU}$ at the distances of the systems. We also recovered nine already known binaries. Ten of our companions have a spectral type of L0 or later, two of them being comfortably in the brown dwarf regime: $2 \mathrm{M} 1115+1607$ (L5 \pm 1$)$ and $2 \mathrm{M} 1259+1001$ (L4.5 \pm 0.5$)$. The latter is a newly identified brown dwarf and orbits a mid-M dwarf. The most widely separated system is NLTT 687, consisting of an M3+L1 pair with a separation of $7400 \mathrm{AU}$. Other very wide systems are TYC 1725-344-1, a G5+M9 pair with a separation of $6700 \mathrm{AU}$ and
LSPMJ1441+1856, a M6+L1 pair with a separation of 4110 AU. Pairs consisting of a G-type star and an ultracool dwarf (e.g., HD21746, G5+M6.5) provide an opportunity to calibrate the metallicity scale of M ultracool dwarfs Rojas-Avala et al. (2010). This calibration is useful because determining the metallicity of such M dwarfs would normally require parallaxes and high-resolution spectra, which are expensive data to acquire and are limited to a few bright cool stars. While our systems are not the most extremes, they can nevertheless help better define the parameter space in which wide low-mass companions can form.

We thank our referee, Eric Mamajek, for excellent suggestions that improved the quality of this paper. This research has made use of the SIMBAD database, operated at CDS, Strasbourg, France and of the VizieR catalogue access tool, CDS, Strasbourg, France. This publication makes use of data products from the Two Micron All Sky Survey, which is a joint project of the University of Massachusetts and the Infrared Processing and Analysis Center/California Institute of Technology, funded by the National Aeronautics and Space Administration and the National Science Foundation. This publication makes use of data products from the Wide-field Infrared Survey Explorer, which is a joint project of the University of California, Los Angeles, and the Jet Propulsion Laboratory/California Institute of Technology, funded by the National Aeronautics and Space Administration. This research has benefitted from the M, L, T, and Y dwarf compendium housed at DwarfArchives.org. This publication has made use of the Very-Low-Mass Binaries Archive housed at http://www.vlmbinaries.org and maintained by Nick Siegler, Chris Gelino, and Adam Burgasser. This research has benefitted from the SpeX Prism Spectral Libraries, maintained by Adam Burgasser at http://pono. ucsd.edu/ adam/browndwarfs/spexprism.

\section{REFERENCES}

Adelman-McCarthy, J. K., Agüeros, M. A., Allam, S. S., et al. 2008, The Astrophysical Journal Supplement Series, 175,297

Ahn, C. P., Alexandroff, R., Allende Prieto, C., et al. 2012, The Astrophysical Journal Supplement Series, 203, 21

Albert, L. 2006, Ph.D. Thesis, 13

Allard, F. 2014, in , 271-272, 00000 
Allers, K. N., \& Liu, M. C. 2013, The Astrophysical Journal, 772,79

Ammons, S. M., Robinson, S. E., Strader, J., et al. 2006, The Astrophysical Journal, 638, 1004

Anderson, E., \& Francis, C. 2013, VizieR Online Data Catalog, 5137, 0

Artigau, E., Doyon, R., Vallee, P., Riopel, M., \& Nadeau, D. 2004, in , 1479-1486

Artigau, É., Lafrenière, D., Doyon, R., et al. 2007, The Astrophysical Journal Letters, 659, L49

Artigau, É., Lafrenière, D., Doyon, R., et al. 2009, in American Institute of Physics Conference Series, Vol. 1094, 15th Cambridge Workshop on Cool Stars, Stellar Systems, and the Sun, ed. E. Stempels, 493-496

Barney, I. 1951, Transactions of the Astronomical Observatory of Yale University, 23, 1, 00000

Bochanski, J. J., Hawley, S. L., \& West, A. A. 2011, The Astronomical Journal, 141, 98

Bonfils, X., Delfosse, X., Udry, S., et al. 2005, Astronomy and Astrophysics, 442, 635

Burgasser, A. J., Kirkpatrick, J. D., \& Lowrance, P. J. 2005, The Astronomical Journal, 129, 2849

Burgasser, A. J., Luk, C., Dhital, S., et al. 2012, The Astrophysical Journal, 757, 110

Close, L. M., Richer, H. B., \& Crabtree, D. R. 1990, The Astronomical Journal, 100, 1968

Cruz, K. L., \& Reid, I. N. 2002, The Astronomical Journal, 123,2828

Cruz, K. L., Reid, I. N., Kirkpatrick, J. D., et al. 2007, The Astronomical Journal, 133, 439

Cushing, M. C., Rayner, J. T., \& Vacca, W. D. 2005, 623, 1115

Cutri, R. M., \& et al. 2012, VizieR Online Data Catalog, 2311,0

Cutri, R. M., Skrutskie, M. F., van Dyk, S., et al. 2003, VizieR Online Data Catalog, 2246, 0

Deacon, N. R., Liu, M. C., Magnier, E. A., et al. 2014, ArXiv e-prints, arXiv:1407.2938

Delorme, P., Delfosse, X., Albert, L., et al. 2008, Astronomy and Astrophysics, 482, 961, 00101

Dhital, S., West, A. A., Stassun, K. G., \& Bochanski, J. J. 2010, The Astronomical Journal, 139, 2566

Dhital, S., West, A. A., Stassun, K. G., et al. 2012, The Astronomical Journal, 143, 67

Duchêne, G., \& Kraus, A. 2013, arXiv:1303.3028 [astro-ph]

Dupuy, T. J., \& Liu, M. C. 2012, The Astrophysical Journal Supplement Series, 201, 19

Epchtein, N., Deul, E., Derriere, S., et al. 1999, Astronomy and Astrophysics, 349, 236

Faherty, J. K., Burgasser, A. J., Bochanski, J. J., et al. 2011, The Astronomical Journal, 141, 71

Faherty, J. K., Burgasser, A. J., West, A. A., et al. 2010, The Astronomical Journal, 139, 176
Fischer, D. A., \& Marcy, G. W. 1992, The Astrophysical Journal, 396, 178

Gagné, J., Lafrenière, D., Doyon, R., Malo, L., \& Artigau, É. 2014, ApJ, 783, 121

Gomes, J. I., Pinfield, D. J., Marocco, F., et al. 2013, Monthly Notices of the Royal Astronomical Society, 431, 2745

Hamuy, M., Suntzeff, N. B., Heathcote, S. R., et al. 1994 , Publications of the Astronomical Society of the Pacific, 106,566

Hawley, S. L., Covey, K. R., Knapp, G. R., et al. 2002, The Astronomical Journal, 123, 3409

Henry, T. J., \& McCarthy, Jr., D. W. 1993, AJ, 106, 773

Hook, I. M., Jørgensen, I., Allington-Smith, J. R., et al. 2004, Publications of the Astronomical Society of the Pacific, 116, 425, 00290

Hrivnak, B. J., Guinan, E. F., \& Lu, W. 1995, The Astrophysical Journal, 455, 300

Janson, M., Hormuth, F., Bergfors, C., et al. 2012, The Astrophysical Journal, 754, 44

Kharchenko, N. V. 2001, Kinematika i Fizika Nebesnykh Tel, 17, 409

Kirkpatrick, J. D., Henry, T. J., \& McCarthy, D. W. 1991 The Astrophysical Journal Supplement Series, 77, 417

Kirkpatrick, J. D., Reid, I. N., Liebert, J., et al. 2000, AJ, 120,447

Lawrence, A., Warren, S. J., Almaini, O., et al. 2007, Monthly Notices of the Royal Astronomical Society, 379, 1599

Lépine, S. 2005, The Astronomical Journal, 130, 1680

Lépine, S., Rich, R. M., \& Shara, M. M. 2003, The Astronomical Journal, 125, 1598

—. 2007, The Astrophysical Journal, 669, 1235

Martín, E. L., Delfosse, X., Basri, G., et al. 1999, The Astronomical Journal, 118, 2466

Martin, E. L., Rebolo, R., \& Zapatero-Osorio, M. R. 1996, The Astrophysical Journal, 469, 706

Mason, B. D., Wycoff, G. L., Hartkopf, W. I., Douglass, G. G., \& Worley, C. E. 2001a, The Astronomical Journal, 122,3466

—. 2001b, The Astronomical Journal, 122, 3466

Massey, P., Strobel, K., Barnes, J. V., \& Anderson, E. 1988, The Astrophysical Journal, 328, 315

Metchev, S. A., \& Hillenbrand, L. A. 2004, in , 238

Meyer, M. R., Adams, F. C., Hillenbrand, L. A., Carpenter, J. M., \& Larson, R. B. 2000, Protostars and Planets IV, 121

Mikami, T., \& Heck, A. 1982, Publications of the Astronomical Society of Japan, 34, 529

Pecaut, M. J., \& Mamajek, E. E. 2013, ApJS, 208, 9

Phan-Bao, N., Crifo, F., Delfosse, X., et al. 2003, Astronomy and Astrophysics, 401, 959 
Phan-Bao, N., Bessell, M. S., Martín, E. L., et al. 2008, Monthly Notices of the Royal Astronomical Society, 383, 831

Pickles, A., \& Depagne, É. 2010, Publications of the Astronomical Society of the Pacific, 122, 1437

Pickles, A. J. 1998, Publications of the Astronomical Society of the Pacific, 110, 863

Porto de Mello, G. F., da Silva, R., da Silva, L., \& de Nader, R. V. 2014, Astronomy \& Astrophysics, 563, A52

Radigan, J., Lafrenière, D., Jayawardhana, R., \& Doyon, R. 2009, The Astrophysical Journal, 698, 405

Raghavan, D., McAlister, H. A., Henry, T. J., et al. 2010, The Astrophysical Journal Supplement Series, 190, 1

Rayner, J. T., Cushing, M. C., \& Vacca, W. D. 2009, The Astrophysical Journal Supplement Series, 185, 289

Reid, I. N., Cruz, K. L., Kirkpatrick, J. D., et al. 2008, AJ, 136,1290

Reid, I. N., \& Gizis, J. E. 1997, The Astronomical Journal, 114, 1992

Reid, I. N., Gizis, J. E., Kirkpatrick, J. D., \& Koerner, D. W. 2001, The Astronomical Journal, 121, 489

Robin, A. C., Reylé, C., Derrière, S., \& Picaud, S. 2003, Astronomy and Astrophysics, 409, 523

Rojas-Ayala, B., Covey, K. R., Muirhead, P. S., \& Lloyd, J. P. 2010, The Astrophysical Journal Letters, 720, L113

Rousselot, P., Lidman, C., Cuby, J.-G., Moreels, G., \& Monnet, G. 2000, Astronomy and Astrophysics, 354, 1134

Salim, S., \& Gould, A. 2003, The Astrophysical Journal, 582,1011

Schmidt, S. J., West, A. A., Hawley, S. L., \& Pineda, J. S. 2010, The Astronomical Journal, 139, 1808

Sheppard, S. S., \& Cushing, M. C. 2009, The Astronomical Journal, 137, 304

Weinberg, M. D., Shapiro, S. L., \& Wasserman, I. 1987, The Astrophysical Journal, 312, 367

West, A. A., Hawley, S. L., Bochanski, J. J., et al. 2008, The Astronomical Journal, 135, 785

Whitworth, A. P., \& Stamatellos, D. 2006, Astronomy and Astrophysics, 458, 817

Wilson, J. C., Kirkpatrick, J. D., Gizis, J. E., et al. 2001, The Astronomical Journal, 122, 1989

Zacharias, N., Monet, D. G., Levine, S. E., et al. 2004, 36, 1418

Zhang, Z. H., Pokorny, R. S., Jones, H. R. A., et al. 2009a, Astronomy and Astrophysics, 497, 619

-. 2009b, Astronomy and Astrophysics, 497, 619

Zhang, Z. H., Pinfield, D. J., Day-Jones, A. C., et al. 2010, Monthly Notices of the Royal Astronomical Society, 404, 1817

This 2-column preprint was prepared with the AAS LATEX macros $\mathrm{v} 5.2$. 